\title{
The Neocortical Progenitor Specification Program Is Established through Combined Modulation of SHH and FGF Signaling
}

\author{
${ }^{\circledR}$ Odessa R. Yabut, ${ }^{1}$ Hui-Xuan Ng, ${ }^{1}$ Keejung Yoon, ${ }^{1,2}$ Jessica C. Arela, ${ }^{1}$ Thomas Ngo, $^{3}$ and ${ }^{\circledR}$ Samuel J. Pleasure ${ }^{1,4}$ \\ ${ }^{1}$ Department of Neurology, University of California San Francisco, San Francisco, California 94158, ${ }^{2}$ College of Biotechnology and Bioengineering, \\ Sungkyunkwan University, Gyeonggi-do 440-746, Suwon, Republic of Korea, ${ }^{3}$ Department of Psychiatry, University of California San Francisco, San \\ Francisco, California 94143, and ${ }^{4}$ Programs in Neuroscience and Developmental Biology, Eli and Edythe Broad Center of Regeneration Medicine \\ and Stem Cell Research, University of California San Francisco, San Francisco, California 94143
}

Neuronal progenitors in the developing forebrain undergo dynamic competence states to ensure timely generation of specific excitatory and inhibitory neuronal subtypes from distinct neurogenic niches of the dorsal and ventral forebrain, respectively. Here we show evidence of progenitor plasticity when Sonic hedgehog $(\mathrm{SHH})$ signaling is left unmodulated in the embryonic neocortex of the mammalian dorsal forebrain. We found that, at early stages of corticogenesis, loss of Suppressor of Fused (Sufu), a potent inhibitor of SHH signaling, in neocortical progenitors, altered the transcriptomic landscape of male mouse embryos. Ectopic activation of SHH signaling occurred, via degradation of Gli3R, resulting in significant upregulation of fibroblast growth factor 15 (FGF15) gene expression in all E12.5 Sufu-cKO neocortex regardless of sex. Consequently, activation of FGF signaling, and its downstream effector the MAPK signaling, facilitated expression of genes characteristic of ventral forebrain progenitors. Our studies identify the importance of modulating extrinsic niche signals such as SHH and FGF15, to maintain the competency and specification program of neocortical progenitors throughout corticogenesis.

Key words: corticogenesis; FGF; lineage fates; neural progenitors; neurogenesis; SHH

Significance Statement

Low levels of FGF15 control progenitor proliferation and differentiation during neocortical development, but little is known on how FGF15 expression is maintained. Our studies identified SHH signaling as a critical activator of FGF15 expression during corticogenesis. We found that Sufu, via Gli3R, ensured low levels of FGF15 was expressed to prevent abnormal specification of neocortical progenitors. These studies advance our knowledge on the molecular mechanisms guiding the generation of specific neocortical neuronal lineages, their implications in neurodevelopmental diseases, and may guide future studies on how progenitor cells may be used for brain repair.

\section{Introduction}

The adult mammalian neocortex is composed of an intricate network of diverse excitatory and inhibitory neurons derived from distinct progenitor domains of the embryonic forebrain. Excitatory

Received Dec. 3, 2019; revised June 22, 2020; accepted July 18, 2020.

Author contributions: 0.R.Y., H.-X.N., K.Y., and S.J.P. designed research; 0.R.Y., H.-X.N., K.Y., J.C.A., and T.N. performed research; 0.R.Y., H.-X.N., K.Y., J.C.A., T.N., and S.J.P. analyzed data; 0.R.Y. and S.J.P. wrote the first draft of the paper; 0.R.Y. and S.J.P. edited the paper; 0.R.Y. and S.J.P. wrote the paper.

The authors declare no competing financial interests.

This work was supported by National Institutes of Health Grants R01s MH077694 and NS118995 to S.J.P., and National Institutes of Health/National Cancer Institute K01CA201068 and American Brain Tumor Association Grant \#ARC1800003 to 0.R.Y. and KNRF 2019M3A9H1103702 to K.Y. We thank members of the S. J.P. laboratory for helpful discussions; Dr. Kenneth Campbell for the Gsx2 antibody; and DeLaine Larsen and Kari Harrington (University of California San Francisco Nikon Imaging Center) for assistance with imaging.

Correspondence should be addressed to Samuel J. Pleasure at sam.pleasure@ucsf.edu.

https://doi.org/10.1523/JNEUROSCI.2888-19.2020

Copyright $\odot 2020$ the authors neurons originate from the ventricular zones (VZs) and subventricular zones (SVZs) of the embryonic neocortex, while inhibitory neurons (interneurons) originate from the ganglionic eminences (GEs). During corticogenesis, radial glial (RG) progenitors populating the VZ/SVZ sequentially generate deep-layer excitatory neurons, followed by upper-layer excitatory neurons via intermediate progenitor cell (IPC) or outer RG cells (Beattie and Hippenmeyer, 2017). This process must be tightly regulated since an imbalance between excitatory and inhibitory activity underlies a number of neurologic and neuropsychiatric disorders (Sohal and Rubenstein, 2019).

A combination of intrinsic and extrinsic cues guide and maintain the specification program of neocortical progenitors throughout corticogenesis to generate neuronal diversity. But the molecular factors integrating these cues in neocortical progenitors to produce distinct neuronal subtypes in a temporal manner are still largely unclear. Our previous work identified 
fundamental mechanisms at early stages of corticogenesis ensuring proper specification of neocortical progenitors into distinct excitatory neuronal lineages, through modulation of Sonic hedgehog (SHH) signaling pathway (Yabut et al., 2015). SHH signaling is triggered on binding of $\mathrm{SHH}$ ligands to the transmembrane receptor Patched 1 (Ptch1), which relieves its inhibition of another transmembrane protein, Smoothened (Smo). Consequently, Smo initiates a cascade of intracellular events promoting the nuclear translocation of Gli, a family transcription factor, to activate $\mathrm{SHH}$ target gene expression. However, intracellular checkpoints are present to modulate $\mathrm{SHH}$ signaling. In the developing neocortex, Suppressor of Fused (Sufu), a potent inhibitor of $\mathrm{SHH}$ signaling, is highly expressed in neocortical progenitors modulating $\mathrm{SHH}$ signals to ensure the production of molecularly distinct upper and deep layer excitatory neurons (Yabut et al., 2015). SUFU exerted this effect by ensuring the stable formation of Gli transcription factors, the downstream effectors of SHH signaling. Specifically, loss of SUFU resulted in the degradation of the repressor form of Gli3 (Gli3R), the predominant Gli protein in the developing neocortex (Palma and Ruiz i Altaba, 2004; Fotaki et al., 2006; H. Wang et al., 2011; Wilson et al., 2012), leading to the production of misspecified neocortical progenitors by mid-corticogenesis. However, little is known on the identity of downstream molecular targets of $\mathrm{SHH}$ signaling or Gli3 in neocortical progenitors, and how deregulation of these targets because of uncontrolled $\mathrm{SHH}$ signaling might affect neocortical progenitor fates.

Here we show that endogenous levels of SHH, in the absence of Sufu, can sufficiently increase SHH signal transduction in neocortical progenitors, resulting in drastic changes in the transcriptomic landscape of the VZ/SVZ at early stages of corticogenesis. In accordance to our previous findings, ventral forebrain progenitor gene transcripts are already ectopically expressed in neocortical progenitors of embryonic (E) 12.5 neocortex mice lacking Sufu. Additionally, we find that activation of fibroblast growth factor (FGF) signaling, via the upregulated gene expression of FGF15, leads to the misspecification of progenitors, particularly affecting the production of IPCs. These novel findings reveal how uncontrolled $\mathrm{SHH}$ signaling and its downstream gene targets can redefine progenitor competency in the embryonic neocortex. Further, this underscores the importance of intrinsic cellular responses, via modulatory proteins, such as Sufu, to temporally restrain extrinsic niche signals that can influence progenitor identity and fate.

\section{Materials and Methods}

Animals. Mice carrying the floxed Sufu allele $\left(\mathrm{Sufu}^{\mathrm{fl}}\right)$ were kindly provided by Chi-Chung Hui (University of Toronto) and were genotyped as described previously (Pospisilik et al., 2010). Emx1-cre (stock \#05628), Rosa-AI14 (stock \#007908), and SmoM2 (stock \#005130) mice were obtained from The Jackson Laboratory. Mice with the genotype Emx1-cre;Sufu ${ }^{f l f l}$ mice are hereto referred to as Sufu-cKO mice. Mice designated as controls did not carry the Cre transgene and may have either one of the following genotypes: Sufu ${ }^{f l+}$ or Sufu ${ }^{f l f l}$. All mouse lines were maintained in mixed strains, and analysis included male and female pups from each age group, although sex differences were not included in data reporting. All animal protocols were in accordance to the National Institute of Health regulations and approved by the University of California San Francisco Institutional Animal Care and Use Committee.

RNA-Seq and analysis. The dorsal forebrain was dissected from E12.5 control and Sufu-cKO male littermates ( $n=4$ per group). Total RNA was extracted using RNEasy Mini Kit (QIAGEN) and prepared for RNAseq. RNASeq was conducted by the University of California San Francisco Functional Genomics Core. Barcoded sequencing libraries were generated using the Truseq Stranded mRNA Library Prep Kit (Illumina). Single-end $50 \mathrm{bp}$ reads were sequenced on the HiSeq4000 (Illumina). Sequencing yielded $\sim 343$ million read with an average read depth of 42.9 million reads/sample. Reads were then aligned using STAR_2.4.2a to the mouse genome (Ensembl Mouse GRCm38.78), and those that mapped uniquely to known mRNAs were used to assess differential expression (DE). Final quantification and statistical testing of differentially expressed (adjusted $p<0.05$ ) genes were performed using DESeq2. Gene set enrichment and pathway analysis was conducted using the DAVID Gene Functional Classification Tool (http://david. abcc.ncifcrf.gov) (Huang et al., 2007). Heatmaps represent transformed FPKM values (Transform $1+\log 2(\mathrm{Y})$ ) and plotted using Prism 8.1 (GraphPad). Filtering was applied for gene ontology enrichment analysis by excluding DE genes with very low normalized read counts (FPKM $<100$ ) in both control and mutant samples. Sequencing data are archived in the Gene Expression Omnibus under GEO Accession \# GSE155851.

Immunohistochemistry. Perfusion, dissection, and immunofluorescence staining were conducted according to standard protocols as previously described (Siegenthaler et al., 2009). Briefly, embryonic brain tissues were fixed by direct immersion in $4 \%$ PFA and postnatal brains fixed by intracardial perfusion followed by $2 \mathrm{~h}$ after fixation. Cryostat sections were air-dried and rinsed $3 \times$ in PBS plus $0.2 \%$ Triton before blocking for $1 \mathrm{~h}$ in $10 \%$ normal lamb serum diluted in PBS with $0.2 \%$ Triton to prevent nonspecific binding. A heat-induced antigen retrieval protocol was performed on selective immunohistochemistry using $10 \mu \mathrm{M}$ citric acid at $\mathrm{pH}$ 6.0. Primary antibodies were diluted in $10 \%$ serum diluted in PBS with $0.2 \%$ Triton containing DAPI; sections were incubated in primary antibody overnight at room temperature. The following antibodies were used: rabbit anti-Tbr2 (1:500 dilution; Abcam, \#ab23345), rabbit anti-GSX2 (1:250 dilution; gift from Kenneth Campbell) (Toresson et al., 2000), mouse anti-Olig2 (1:250 dilution; Millipore, \#MABN50), and phosphorylated-Erk1/2 (pERK1/2 1:100 dilution, Cell Signaling Technology, \#4370). To detect primary antibodies, we used species-specific AlexaFluor-conjugated secondary antibodies (1:500; Invitrogen) in $1 \times$ PBS-T for $1 \mathrm{~h}$ at room temperature, washed with $1 \times$ PBS, and coverslipped with Fluoromount-G (Southern Biotechnology).

ISH. Lhx2, CoupTF2 ISH was conducted using RNA probes kindly provided by Professor John Rubenstein (University of California San Francisco). Dlx1 and Dbx1 riboprobes were generated using primer sequences published by the Allen Brain Atlas ISH Database (http:// developingmouse.brain-map.org/) with SP6 and T7 promoter binding sequences included in $5^{\prime}$ ends. Target gene cDNA was amplified from pooled cDNA reactions made from mouse brain; total RNA was used as a template source. DIG-labeled RNA probes were generated using the DIG RNA Labeling Kit SP6/T7 (Sigma Millipore, catalog \#11175025910) according to the manufacturer's protocols. DIG-labeled RNA probes were diluted in hybridization buffer (50\% formamide, $5 \times$ SSC, $0.3 \mathrm{mg} / \mathrm{ml}$ tRNA, $100 \mu \mathrm{l} / \mathrm{ml}$ heparin, $1 \times$ Denhardt's solution, $0.1 \%$ Tween $20,0.1 \%$ CHAPS, 5 mm EDTA) and added to RNase-free cryosections for incubation in a humidified chamber at $65^{\circ} \mathrm{C}$ for $16-20 \mathrm{~h}$. Sections were washed in $0.2 \times$ SSC (Ambion, AM9770) at $65^{\circ} \mathrm{C}$ followed by PBST at room temperature. Tissue sections were incubated in alkaline phosphatase-conjugated anti-DIG antibody (1:1500, Roche Applied Sciences, 11093274910) for 16-20 h incubation at room temperature, and colorimetric signals were detected using NBT/BCIP (Roche Applied Sciences, 11383221001).

RNAScope ISH was conducted for Fgf15 and PTCH1. RNAscope probes Mm-Ptch1 (catalog \#402811) and Mm-FGF15 (catalog \#412811) were designed commercially by the manufacturer (Advanced Cell Diagnostics). RNAScope Assay was performed using the RNAscope Multiplex Fluorescent Reagent Kit V2 according to the manufacturer's instructions. Detection of the probe was done with Opal 570 or Opal 520 reagent (PerkinElmer).

Forebrain organotypic slice culture. Whole brains from E12.5 WT CD-1 mice were carefully dissected and placed in ice-cold HBSS (Invitrogen). Brains were embedded in 4\% Low Melting Point Agarose (Nueve)/HBSS mix and allowed to solidify on ice. Embedded brains were sliced using a VT1000S vibratome (Leica Microsystems) into 400$\mu \mathrm{m}$-thick slices and placed in Recovery Media: MEM (Invitrogen) with Glutamax (Invitrogen) and pennicillin/streptomycin (Invitrogen). Slices 
Table 1. Statistical analyses ${ }^{a}$

\begin{tabular}{|c|c|c|c|c|c|}
\hline Figure & Parameter & Groups & Statistical test & Outcome & $p$ \\
\hline \multirow[t]{5}{*}{$5 D$} & E12.5 phospho-Erk1/2 ${ }^{+}$ & A-P position vs genotype (E12.5 control and Sufu-cKO) & Repeated-measures two-way ANOVA & $F$ position $\times$ genotype $(3,24)=3.329$ & 0.0365 \\
\hline & regions & Control $(n=5)$ vs Sufu-cKO $(n=5)$ Position 1 & Holm-Sidak's multiple comparisons test & $t=3.685, \mathrm{df}=5.547$ & 0.018 \\
\hline & & Control $(n=5)$ vs Sufu-cKO $(n=5)$ Position 2 & & $t=6.861, \mathrm{df}=4.873$ & 0.0033 \\
\hline & & Control $(n=5)$ vs Sufu-cKO $(n=5)$ Position 3 & & $t=8.112, \mathrm{df}=4.949$ & 0.0019 \\
\hline & & Control $(n=5)$ vs Sufu-cKO $(n=5)$ Position 4 & & $t=5.310, \mathrm{df}=4.296$ & 0.0099 \\
\hline \multirow[t]{5}{*}{$5 E$} & E14.5 phospho-Erk1/2 ${ }^{+}$ & A-P position vs genotype (E14.5 control and Sufu-cKO) & Repeated-measures two-way ANOVA & $F$ position vs genotype $(3,19)=4.684$ & 0.013 \\
\hline & regions & Control $(n=4)$ vs Sufu-cKO $(n=3)$ Position 1 & Holm-Sidak's multiple comparisons test & $t=7.255, \mathrm{df}=27$ & $<0.0001$ \\
\hline & & Control $(n=5)$ vs Sufu-cKO $(n=5)$ Position 2 & & $t=5.452, \mathrm{df}=27$ & $<0.0001$ \\
\hline & & Control $(n=5)$ vs Sufu-cKO $(n=5)$ Position 3 & & $t=4.665, \mathrm{df}=27$ & 0.0001 \\
\hline & & Control $(n=4)$ vs Sufu-cKO $(n=4)$ Position 4 & & $t=3.67, \mathrm{df}=27$ & 0.0011 \\
\hline \multirow[t]{16}{*}{60} & $\mathrm{Tbr}^{+}$cells & Treatment ( $n=3$ per condition) & One-way ANOVA & $F$ treatment $(5,12)=14.15$ & 0.0001 \\
\hline & & DMSO vs Fgf15 & Holm-Sidak's multiple comparisons test & $t=3.319, \mathrm{df}=12$ & 0.0537 \\
\hline & & DMSO vs Shh & & $t=6.515, \mathrm{df}=12$ & 0.0004 \\
\hline & & DMSO vs Fgf15+Shh & & $t=5.651, \mathrm{df}=12$ & 0.0014 \\
\hline & & DMSO vs Cyclo & & $t=0.5662, \mathrm{df}=12$ & 0.6585 \\
\hline & & DMSO vs Cyclo + Fgf15 & & $t=4.4, \mathrm{df}=12$ & 0.0095 \\
\hline & & Fgf15 vs Shh & & $t=3.196, \mathrm{df}=12$ & 0.0599 \\
\hline & & Fgf15 vs Fgf15+Shh & & $t=2.332, \mathrm{df}=12$ & 0.2072 \\
\hline & & Fgf15 vs Cyclo & & $t=2.753, \mathrm{df}=12$ & 0.1163 \\
\hline & & Fgf15 vs Cyclo + Fgf15 & & $t=1.081, \mathrm{df}=12$ & 0.6585 \\
\hline & & Shh vs Fgf15+Shh & & $t=0.8646, \mathrm{df}=12$ & 0.6585 \\
\hline & & Shh vs Cyclo & & $t=5.949, \mathrm{df}=12$ & 0.0009 \\
\hline & & Shh vs Cyclo + Fgf15 & & $t=2.115, \mathrm{df}=12$ & 0.2503 \\
\hline & & Fgf15+ Shh vs Cyclo & & $t=5.085, \mathrm{df}=12$ & 0.0032 \\
\hline & & Fgf15+ Shh vs Cyclo+Fgf15 & & $t=1.251, \mathrm{df}=12$ & 0.6572 \\
\hline & & Cyclo vs Cyclo + Fgf15 & & $t=3.834, \mathrm{df}=12$ & 0.0235 \\
\hline 71 & $\mathrm{Tbr}^{+}$cells & Treatment: DMSO $(n=6)$ vs FGF15 $(n=6)$ & Unpaired $t$ test (two-tailed) & $t=2.805, \mathrm{df}=10$ & 0.0186 \\
\hline
\end{tabular}

${ }^{a}$ Summary of statistical analyses performed for Figures 5-7.

were transferred into uncoated Millicell-CM membrane inserts (EMD Millipore) in 6-well plates (BD Biosciences) and cultured in Neurobasal (Invitrogen) supplemented with Glutamax (Invitrogen), pennicillin/ streptomycin (Invitrogen), B-27 (Invitrogen), and N2 (Invitrogen) at $37^{\circ} \mathrm{C}, 5 \% \mathrm{CO}_{2}$, and $100 \%$ humidity. After 2 DIV, cell culture media were aspirated, and slices were washed in $1 \times$ PBS, fixed in cold $4 \%$ PFA for 30 min, cryoprotected in $30 \%$ sucrose, and embedded in OCT. Slices were cryosectioned into $20-\mu \mathrm{m}$-thick coronal sections and stored at $-80^{\circ} \mathrm{C}$ until used for immunofluorescence analysis as described above. Treatment (as described in text) of organotypic slices was conducted 2-3 $\mathrm{h}$ after initial plating and incubation of slices with the following concentrations: $100 \mathrm{ng} / \mathrm{ml}$ recombinant FGF15 (Prospec Bio, \#CYT-027), $200 \mathrm{ng} / \mathrm{ml}$ recombinant SHH (GenScript, \#Z03050-50), and $5 \mu \mathrm{M}$ cyclopamine (Toronto Research Chemicals, \#C988400). Following treatments, slice cultures were incubated for $2 \mathrm{~d}$ and processed as described above.

Image analysis and acquisition. Images were acquired using a Nikon EDC microscope equipped with a QCapture Pro camera (QImaging), Axioscan Z.1 (Carl Zeiss) using the Zen 2 blue edition software (Carl Zeiss), or the Nikon Ti inverted microscope with CSU-W1 large FOV confocal and Andor Zyla 4.2 sCMOS camera. All images were imported in tiff or jpeg format. Brightness, contrast, and background were adjusted equally for the entire image between controls and mutant using the "Brightness/Contrast" and "Levels" function from "Image/ Adjustment" options in Adobe Photoshop or National Institutes of Health ImageJ without any further modification. National Institutes of Health ImageJ was used to threshold background levels between controls and mutant tissues to quantify fluorescence labeling. For pErk1/2 quantification, the total area with positive pErk1/2 labeling was measured, which began in the pallial-subpallial boundary in the controls and extended dorsally in Sufu-cKO neocortex, for each hemisphere across the anterior to posterior
A

E12.5

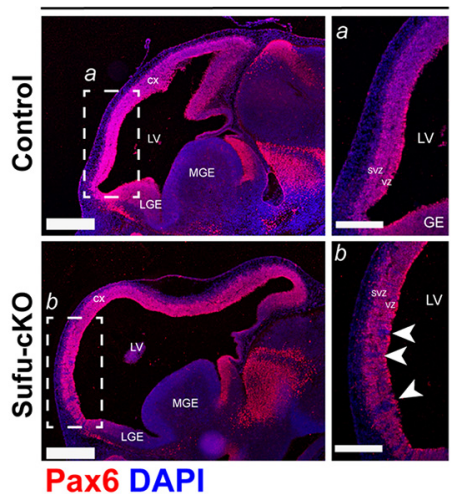

B

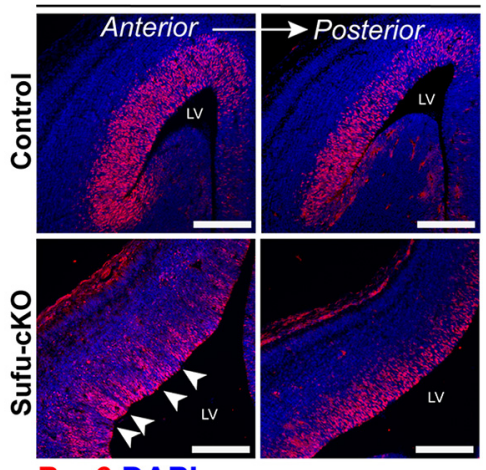

Pax6 DAPI

Figure 1. Neocortical progenitor defects are evident in discrete regions in the E12.5 Sufu-cK0 neocortex. $\boldsymbol{A}$, Immunofluorescence staining of sagittal sections of E12.5 control and Sufu-cKO embryonic forebrains, using dorsal forebrain progenitor marker, Pax6, and DAPI counterstain, shows high Pax6 expression in the dorsal forebrain (CX) along the lateral ventricles (LV) compared with the lateral (LGE) or medial (MGE) GE in both genotypes. $\boldsymbol{A}, \boldsymbol{B}$, Higher magnification of boxed regions represents low or absent Pax6 expression in specific areas of the anterior neocortex of Sufu-cKO forebrains (arrows, $b$ ) particularly in the VZ and SVZ but not in controls (a). These defects were not evident in the E10.5 Sufu-cKO or control forebrains (Extended Data Fig. 1-1). Sections are counterstained with DAPI. Scale bars: $\boldsymbol{A}, \boldsymbol{B} 500 \mu \mathrm{m} ; \boldsymbol{a}, \boldsymbol{b}, 250 \mu \mathrm{m}$. $\boldsymbol{B}$, Pax6 immunofluorescence staining of coronal sections of the E14.5 control and Sufu-cK0 represents areas lacking Pax6 expression in the anterior neocortex of the E14.5 SufucKO mice (arrows) but not in posterior regions or in controls. Scale bar, $200 \mu \mathrm{m}$.

axis. One forebrain section in each representative anterior to posterior region was measured (see Fig. 5C) from both hemispheres and averaged. All analyses were conducted in at least two or three 20 - $\mu$ m-thick sections that were histologically matched at the rostral-caudal level between genotypes.

Statistics. Prism 8.1 (GraphPad) was used for statistical analysis. Two-sample experiments were analyzed by Student's $t$ test, and experiments with more than two parameters were analyzed by ANOVA. In 
A

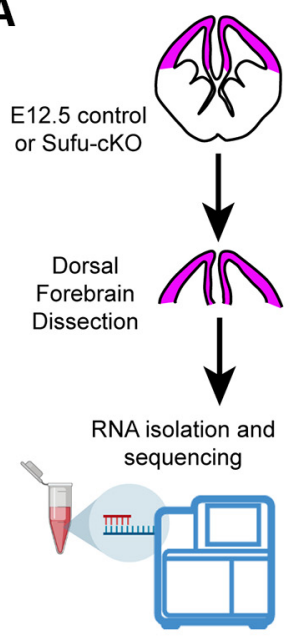

Control

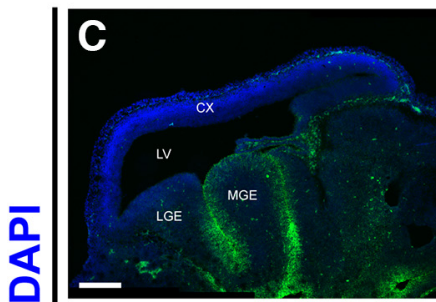

든

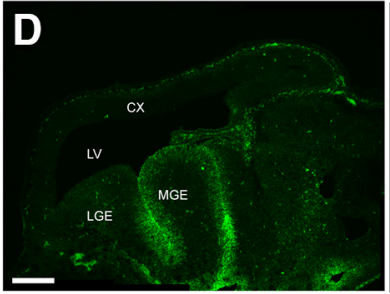

B

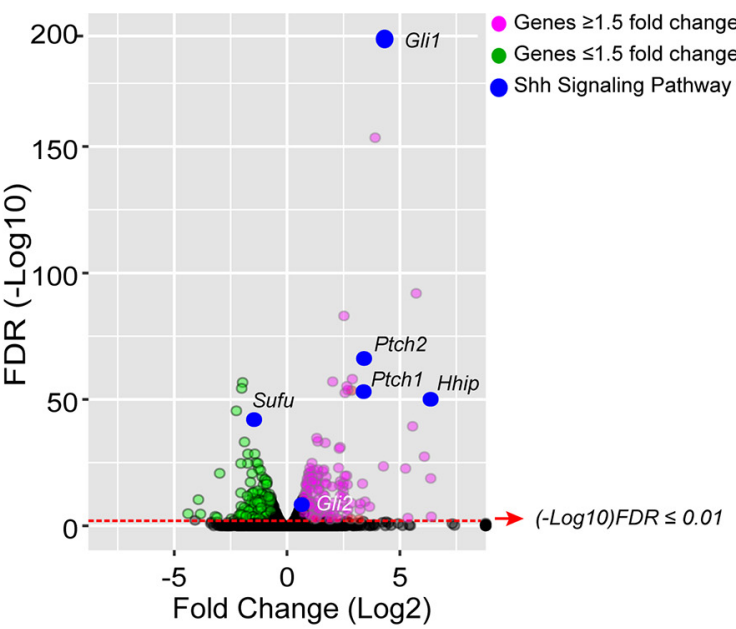

Sufu-ckO
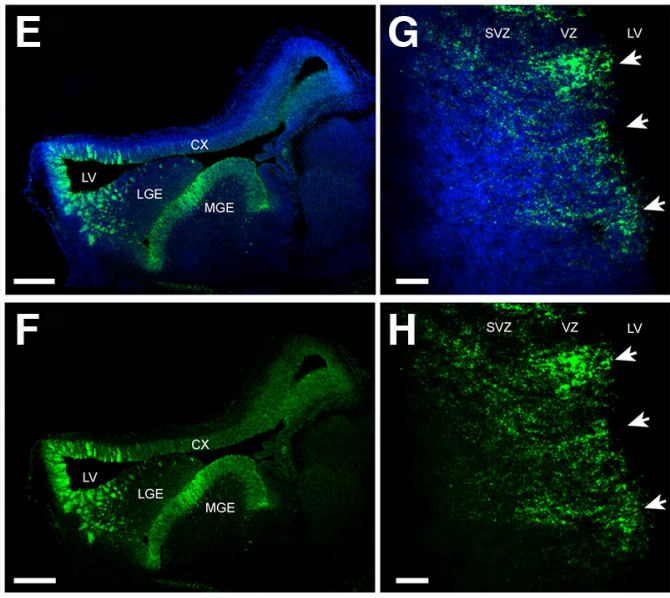

Figure 2. Upregulated expression of SHH signaling gene targets in neocortical progenitors of the E12.5 Sufu-cKO dorsal forebrain. $A$, Schematic showing dorsal forebrain areas (pink) dissected from control and mutant E12.5 mice for RNA-Seq analysis. $\boldsymbol{B}$, Volcano plot of RNA-Seq dataset highlighting differentially expressed genes with adjusted $p$ value $<0.01$ (FDR $(-\log 10))$ and fold change $(\log 2) \geq 1.5$ (red circles) or fold change $(\log 2) \leq 1.5$ (green circles), and genes in the SHH signaling pathway (blue circles), between the E12.5 dorsal forebrain of controls and Sufu-cKO E12.5 embryos (see also Tables 2 and 3). $\mathbf{C}-\boldsymbol{F}$, RNAscope ISH on sagittal brain sections using probes for Patched 1 (Ptch1) validates upregulation of Ptch1 RNA expression in the E12.5 Sufu-cKO dorsal forebrain $(\boldsymbol{E}, \boldsymbol{F})$, whereas Ptch1 RNA expression is only detected in the MGE of controls $(\boldsymbol{C}, \boldsymbol{D}) . \boldsymbol{C}, \boldsymbol{E}$, Sections are counterstained with DAPI. Scale bar, $500 \mu \mathrm{m} . \boldsymbol{G}, \boldsymbol{H}$, Higher magnification of rostral neocortex of E12.5 Sufu-cKO dorsal forebrain showing that Ptch1 RNA expression is preferentially higher along the VZ and SVZ where neocortical progenitors are localized. Ptch1 expression also appear in columns, radiating inward from the apical VZ (arrows). G, Sections are counterstained with DAPI. Scale bar, $25 \mu \mathrm{m}$.

one- or two-way ANOVA, when interactions were found, follow-up analyses were conducted for the relevant variables using Holm-Sidak's multiple comparisons test. All experiments were conducted at least in triplicate with sample sizes of $n=3-6$ embryos/animals/slices per genotype. A $p$ value $\leq 0.05$ was considered statistically significant. Graphs represent mean \pm SEM. Statistical values and analyses are summarized in Table 1.

\section{Results}

Specification defects are evident in discrete regions of the neocortex of E12.5 embryonic mice lacking Sufu

The role of SHH signaling in neocortical neuron specification is critical before E13.5, a time point at which superficial projection neurons are just beginning to differentiate. Analysis of mice, in which Sufu is conditionally deleted at E10.5 in neocortical progenitors using the
Emx1-Cre driver (Emx1-cre/+;Sufu-fllfl or Sufu-cKO), revealed that modulating SHH signaling is critical to properly specify distinct superficial and deep layer projection neurons, after dorsoventral patterning of the forebrain (Yabut et al., 2015). While specification defects were clear at E14.5 in Sufu-cKO cortex, any molecular changes before this time point were not deeply examined in the previous study. Since changes in Gli2 and Gli3R levels were apparent at E12.5, we postulated that critical molecular alterations must have occurred at this time point. We therefore initiated our studies by careful examination of Pax6 expression, which is highly expressed in neocortical RG progenitors (Ypsilanti and Rubenstein, 2016). As expected, we found that Pax6 exclusively expressed in dorsal forebrain regions of the E12.5 control and Sufu-cKO brains, and not in the GE (Fig. 1A). However, Pax6 expression was noticeably intermittent in anterior regions of the E12.5 Sufu-cKO neocortex (Fig. $1 \mathrm{Ab}$, boxed regions, arrowheads). Moreover, these Pax6-negative areas exhibited a columnar distribution hinting at anomalous RG clones. Analysis of corresponding regions showed that the E14.5 Sufu-cKO neocortex similarly displayed columnar distribution of $\mathrm{Pax}^{+}$ and $\mathrm{Pax6}^{-}$regions in anterior regions (Fig. $1 B$, arrowheads), but this distribution was not prevalent in posterior regions (Fig. 1B). These defects were not present at E10.5, in which the distribution of $\mathrm{Pax}^{+}$cells were largely indistinguishable between controls and Sufu-cKO embryos (Extended Data Fig. 1-1). Therefore, despite having properly formed dorsal forebrain domains, a subpopulation of neocortical RG progenitors displayed aberrant behavior in the E12.5 Sufu-cKO neocortex.

\section{Upregulated expression of SHH signaling targets in Sufu mutant neocortical progenitors}

To better understand the molecular changes in neocortical progenitors of the E12.5 Sufu-cKO neocortex, we isolated total RNA from dissected control and mutant dorsal forebrain for transcriptome profiling by RNA-Seq (Fig. 2A). Sequencing yielded $\sim 343$ million reads with an average read depth of 42.9 million reads/sample ( $n=4$ per genotype). Reads were then aligned to the mouse genome, and we successfully mapped $98.69 \pm 0.04 \%$ of the fragments to the genome, with $17.01 \pm$ $0.11 \%$ of fragments mapped to multiple locations of the genome. Those that mapped uniquely to known mRNAs $(81.68 \pm 0.12 \%$ of fragments) were used to assess DE. Differentially expressed genes (with average FPKM $>20$ in at least one sample/genotype group) were assessed (Table 2).

We confirmed that SHH signaling gene targets, such as Gli1, Patched 1 and 2 (Ptch1 and Ptch2), and the Hedgehog-Interacting 
Table 2. Top differentially expressed genes between E12.5 control and Sufu-cKO dorsal forebrain ${ }^{a}$

\begin{tabular}{|c|c|c|c|c|c|c|c|c|c|}
\hline \multirow[b]{2}{*}{ Ensembl_ID } & \multirow[b]{2}{*}{ Gene } & \multicolumn{4}{|c|}{ Mutant vs control } & \multicolumn{2}{|l|}{ Controls (FPKM) } & \multicolumn{2}{|l|}{ Sufu-cKO (FPKM) } \\
\hline & & $\mathrm{FC}$ & $\log 2 \mathrm{FC}$ & RawP & FDR & Average & SD & Average & SD \\
\hline ENSMUSG00000075707 & Dio3 & 82.3875172 & 6.36435386 & $4.7499 \mathrm{E}-22$ & $1.7416 \mathrm{E}-19$ & 7.96831358 & 4.59579515 & 656.489572 & 229.835653 \\
\hline ENSMUSG00000097758 & Dio3os & 69.4175062 & 6.11722763 & $1.4018 \mathrm{E}-41$ & $1.612 \mathrm{E}-38$ & 5.84745817 & 5.83167716 & 405.915963 & 93.8868307 \\
\hline ENSMUSG00000032517 & Mobp & 67.8055024 & 6.08333045 & $5.5873 \mathrm{E}-31$ & $4.2835 \mathrm{E}-28$ & 1.86323925 & 2.34119106 & 126.337874 & 31.3170661 \\
\hline ENSMUSG00000031073 & Fgf15 & 52.7038764 & 5.71983717 & $1.3756 \mathrm{E}-96$ & $1.16 \mathrm{E}-92$ & 152.277335 & 111.900656 & 8025.60586 & 1237.95433 \\
\hline ENSMUSG00000030507 & Dbx1 & 38.0194446 & 5.24866555 & $3.6317 \mathrm{E}-26$ & $2.0881 \mathrm{E}-23$ & 27.7421756 & 13.2249167 & 1054.74211 & 301.451019 \\
\hline ENSMUSG00000040543 & Pitpnm3 & 19.2787426 & 4.26893905 & $4.7584 \mathrm{E}-27$ & $3.0096 \mathrm{E}-24$ & 10.5181239 & 1.3609172 & 202.776203 & 48.0779528 \\
\hline ENSMUSG00000025407 & Gli1 & 18.6364862 & 4.22005797 & $3.99 \mathrm{E}-198$ & $1.009 \mathrm{E}-193$ & 81.5909417 & 30.4343144 & 1520.56846 & 123.97597 \\
\hline ENSMUSG00000038156 & Spon1 & 14.9532123 & 3.90238353 & $2.55 \mathrm{E}-158$ & $3.225 \mathrm{E}-154$ & 56.2092056 & 22.9496826 & 840.508183 & 67.3977619 \\
\hline ENSMUSG00000096999 & Gm26793 & 13.902041 & 3.7972248 & $1.3196 \mathrm{E}-66$ & $4.7694 \mathrm{E}-63$ & 15.772933 & 4.61397622 & 219.275962 & 28.1418305 \\
\hline ENSMUSG00000039830 & Olig2 & 12.6102384 & 3.65652365 & $2.65 \mathrm{E}-10$ & $2.7033 \mathrm{E}-08$ & 69.5186604 & 19.2058548 & 876.646882 & 335.706 \\
\hline ENSMUSG00000063600 & Egfem1 & 9.46791651 & 3.24304698 & $1.358 \mathrm{E}-11$ & $1.6759 \mathrm{E}-09$ & 10.8922792 & 6.68726483 & 103.12719 & 32.3185231 \\
\hline ENSMUSG00000046160 & Olig1 & 9.35940117 & 3.22641623 & $4.6615 \mathrm{E}-09$ & $3.905 \mathrm{E}-07$ & 6.21737577 & 1.93182166 & 58.1909141 & 21.2357905 \\
\hline ENSMUSG00000035946 & Gsx2 & 9.19782474 & 3.20129271 & $8.7129 \mathrm{E}-05$ & 0.00271461 & 40.8981257 & 51.8805517 & 376.173792 & 198.118943 \\
\hline ENSMUSG00000085072 & Ict1os & 7.94959453 & 2.99088128 & $2.0916 \mathrm{E}-13$ & $3.4361 \mathrm{E}-11$ & 5.31695799 & 2.76251218 & 42.2676602 & 8.90946841 \\
\hline ENSMUSG00000028370 & Рappa & 7.41766068 & 2.89096427 & $2.9723 \mathrm{E}-62$ & $9.3997 \mathrm{E}-59$ & 293.550189 & 58.6301674 & 2177.45569 & 265.650782 \\
\hline ENSMUSG00000102796 & RP23-335B9.5 & 7.31629286 & 2.87111282 & $4.655 E-11$ & $5.353 \mathrm{E}-09$ & 4.77449345 & 4.42629104 & 34.9315923 & 3.48684994 \\
\hline ENSMUSG00000030862 & Cpxm2 & 7.24779323 & 2.8575418 & $1.7851 \mathrm{E}-57$ & $3.2258 \mathrm{E}-54$ & 39.618948 & 11.0091521 & 287.149943 & 5.16012755 \\
\hline ENSMUSG00000045591 & Olig3 & 7.20471446 & 2.84894125 & $8.3417 \mathrm{E}-12$ & $1.0658 \mathrm{E}-09$ & 5.25145549 & 3.63879005 & 37.8352373 & 3.88669789 \\
\hline ENSMUSG00000003227 & Edar & 7.07128346 & 2.82197209 & $4.5951 \mathrm{E}-05$ & 0.00156886 & 14.2573202 & 6.28628971 & 100.817552 & 49.3383641 \\
\hline ENSMUSG00000036466 & Megf11 & 6.46970608 & 2.69370017 & 5.4537E-09 & $4.4364 \mathrm{E}-07$ & 65.0626058 & 12.0041891 & 420.935937 & 144.39887 \\
\hline ENSMUSG00000074785 & Plxnc1 & 6.4646009 & 2.69256131 & 1.0379E-57 & $2.0198 \mathrm{E}-54$ & 1220.54603 & 85.3997809 & 7890.34299 & 984.760757 \\
\hline ENSMUSG00000099907 & Gm10421 & 6.39502413 & 2.6769498 & 0.00019259 & 0.00527872 & 5.31695799 & 2.76251218 & 34.0020746 & 17.1217016 \\
\hline ENSMUSG00000031075 & Ano1 & 6.35732479 & 2.6684198 & $1.743 \mathrm{E}-19$ & $4.7931 \mathrm{E}-17$ & 195.346022 & 110.705417 & 1241.87811 & 247.642224 \\
\hline ENSMUSG00000036264 & Fstl4 & 5.66355645 & 2.50170828 & $8.1061 \mathrm{E}-10$ & $7.4573 \mathrm{E}-08$ & 7.06205978 & 5.2274176 & 39.9963742 & 10.1087574 \\
\hline ENSMUSG00000027832 & Ptx3 & 5.62037022 & 2.49066516 & $3.7632 \mathrm{E}-20$ & $1.1334 \mathrm{E}-17$ & 323.719795 & 71.905768 & 1819.4251 & 368.556876 \\
\hline ENSMUSG00000025856 & Pdgfa & 5.60936415 & 2.48783724 & $8.4139 \mathrm{E}-17$ & $1.9529 \mathrm{E}-14$ & 213.357738 & 41.0886507 & 1196.80125 & 267.580448 \\
\hline ENSMUSG00000039579 & Grin3a & 5.57374247 & 2.47864634 & $1.1749 \mathrm{E}-12$ & $1.7382 \mathrm{E}-10$ & 23.3137923 & 14.1868649 & 129.945074 & 30.2336844 \\
\hline ENSMUSG00000020902 & Ntn1 & 5.5507006 & 2.47266988 & $1.2616 \mathrm{E}-18$ & $3.1918 \mathrm{E}-16$ & 182.967354 & 114.72434 & 1015.597 & 180.417925 \\
\hline ENSMUSG00000015501 & Hivep2 & 5.36960862 & 2.42481694 & $2.9316 \mathrm{E}-27$ & $1.9017 \mathrm{E}-24$ & 366.270823 & 58.4251106 & 1966.73097 & 333.806373 \\
\hline ENSMUSG00000026765 & Lypd6b & 5.15521956 & 2.36603387 & $7.9847 \mathrm{E}-21$ & $2.658 \mathrm{E}-18$ & 64.128181 & 21.8461262 & 330.594853 & 59.9726836 \\
\hline ENSMUSG00000071862 & Lrrtm2 & 5.15367748 & 2.36560226 & $5.4108 \mathrm{E}-15$ & $1.0573 \mathrm{E}-12$ & 99.5065322 & 21.4481348 & 512.824574 & 118.534936 \\
\hline ENSMUSG00000044499 & Hs3st5 & 5.1458571 & 2.36341139 & 0.00036629 & 0.00909391 & 5.29148754 & 6.35506294 & 27.2292387 & 11.371032 \\
\hline ENSMUSG00000022449 & Adamts20 & 5.09486349 & 2.34904349 & $8.4761 \mathrm{E}-35$ & $7.6584 \mathrm{E}-32$ & 879.123707 & 153.586556 & 4479.01527 & 645.467259 \\
\hline ENSMUSG00000047935 & Gm5607 & 5.07145449 & 2.34239957 & $1.5793 \mathrm{E}-17$ & $3.8051 \mathrm{E}-15$ & 235.233075 & 84.7104464 & 1192.97383 & 238.817078 \\
\hline ENSMUSG00000050511 & Oprd1 & 5.01904508 & 2.32741291 & $3.4705 \mathrm{E}-19$ & $9.0517 \mathrm{E}-17$ & 18.3322572 & 2.46129774 & 92.0104253 & 8.50394075 \\
\hline ENSMUSG00000042942 & Greb1l & 4.97801523 & 2.31557065 & $3.1282 \mathrm{E}-34$ & $2.729 \mathrm{E}-31$ & 40.9347158 & 11.732061 & 203.773639 & 24.7529018 \\
\hline ENSMUSG00000024565 & Sall3 & 4.87269605 & 2.28472023 & $1.9085 \mathrm{E}-26$ & $1.1496 \mathrm{E}-23$ & 323.047376 & 135.817299 & 1574.11167 & 225.027015 \\
\hline ENSMUSG00000050830 & Vwc2 & 4.77175349 & 2.25451952 & $4.5876 \mathrm{E}-10$ & 4.4985E-08 & 15.1718669 & 6.17372207 & 72.3964088 & 19.3261315 \\
\hline ENSMUSG00000079042 & Trim61 & 4.65688672 & 2.21936579 & $2.3428 \mathrm{E}-05$ & 0.00087679 & 13.1499629 & 7.22679895 & 61.2378877 & 23.6763672 \\
\hline ENSMUSG00000020182 & Ddc & 4.23590166 & 2.0826691 & $9.4268 \mathrm{E}-08$ & $5.9622 \mathrm{E}-06$ & 20.3591524 & 11.1225092 & 86.2393673 & 24.3118885 \\
\hline ENSMUSG00000047773 & Ankfn1 & 4.21116097 & 2.07421802 & $1.1688 \mathrm{E}-07$ & 7.1944E-06 & 24.0803028 & 16.5671624 & 101.406031 & 27.6755788 \\
\hline ENSMUSG00000024302 & Dtna & 4.18161594 & 2.06406056 & $5.1632 \mathrm{E}-12$ & $6.8081 \mathrm{E}-10$ & 338.390772 & 40.517148 & 1415.02025 & 341.918631 \\
\hline ENSMUSG00000036800 & Fam135b & 4.12972627 & 2.04604616 & $6.3431 \mathrm{E}-08$ & 4.1682E-06 & 9.42253743 & 4.08437337 & 38.9125003 & 3.17059338 \\
\hline ENSMUSG00000074607 & Tox2 & 4.05513698 & 2.01975065 & 3.3761E-61 & 9.4903E-58 & 214.277748 & 34.3114331 & 868.925619 & 45.6255507 \\
\hline ENSMUSG00000075585 & 6330403L08Rik & 4.04965382 & 2.01779859 & $1.1438 \mathrm{E}-23$ & $4.9045 \mathrm{E}-21$ & 81.839912 & 11.821983 & 331.423312 & 52.4601701 \\
\hline ENSMUSG00000079022 & Col22a1 & 3.99687359 & 1.99887194 & $1.1506 \mathrm{E}-18$ & $2.9403 \mathrm{E}-16$ & 190.333392 & 55.8880568 & 760.738507 & 130.864341 \\
\hline ENSMUSG00000042596 & Tfap2d & 3.97894006 & 1.99238417 & $3.5297 \mathrm{E}-05$ & 0.00124026 & 5.85024556 & 2.80274705 & 23.2777764 & 4.85423132 \\
\hline
\end{tabular}




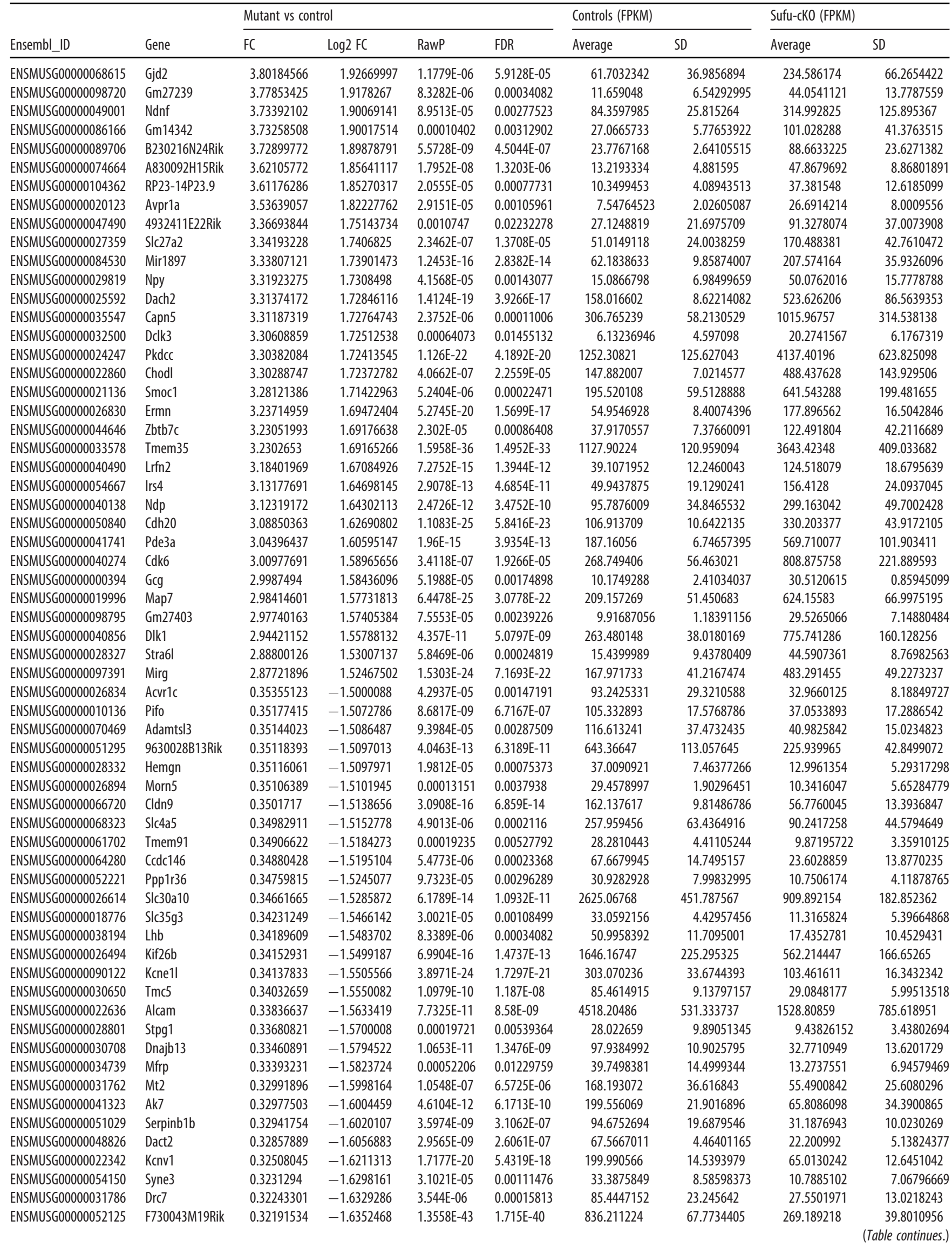




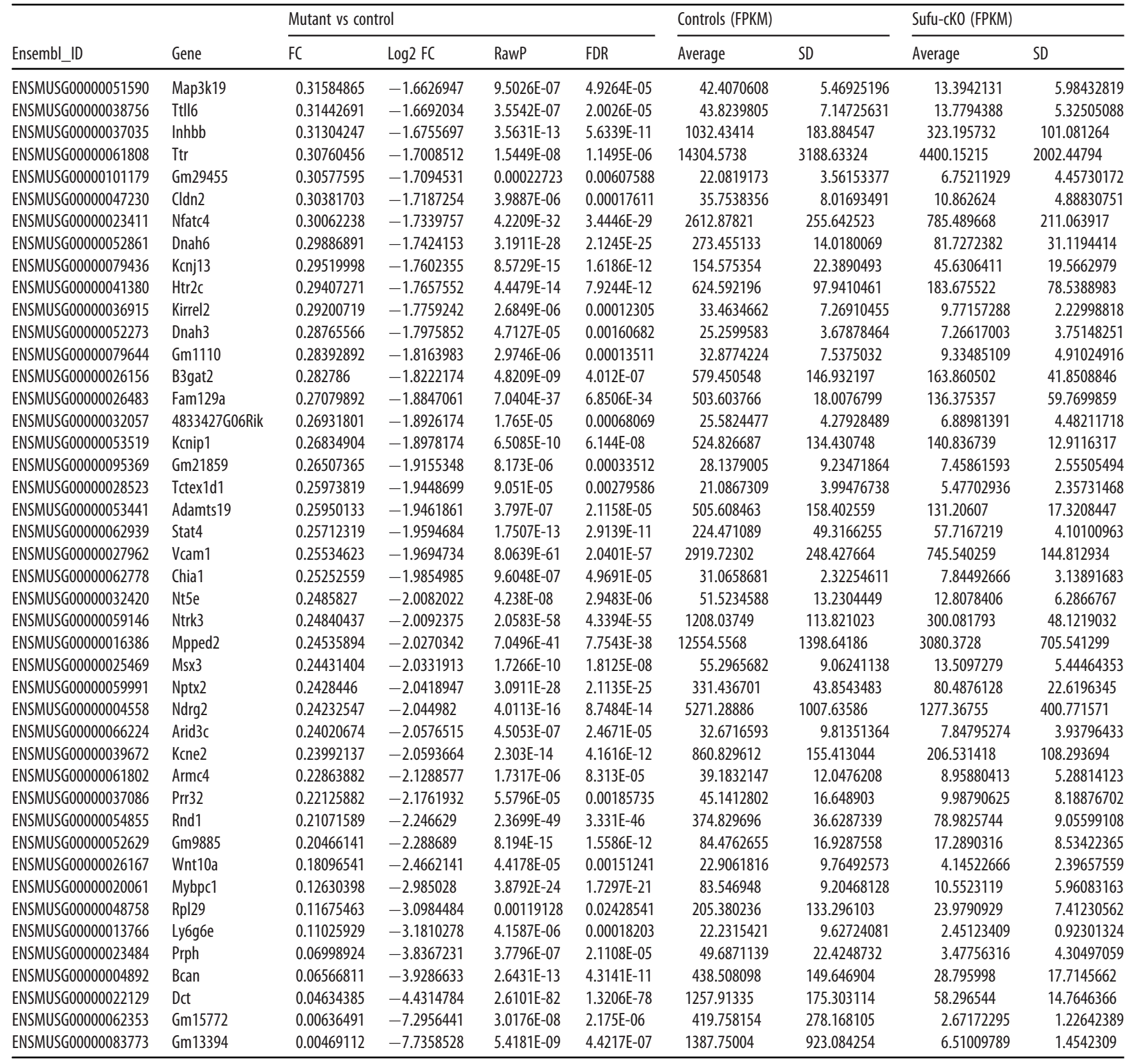

${ }^{a}$ The top significantly expressed genes (FDR $<0.05$ ) with a Log2FC of \pm 1.5 ; and read counts (FPKM) $>20$ for at least 1 genotype group in the E12.5 Sufu-cKO dorsal forebrain $(n=4$ per genotype). FC, Unlogged fold change; Log2FC, $\log 2$ fold change; RawP, unadjusted $p$ value; FDR, $p$ value adjusted for multiple comparisons; average FPKM, average normalized sample values; SD, standard deviation of normalized sample values.

Protein (Hhip), are specifically upregulated in the E12.5 SufucKO neocortex compared with controls (FDR $<0.01$; Fig. $2 B$; Table 3). We validated these observations by ISH using probes for Ptch1, which was ectopically expressed throughout the neocortical expanse (Fig. $2 E, F$ ) in contrast to controls (Fig. 2C,D). Levels of Ptch1 expression were confined within the VZ/SVZ across the cortical and hippocampal primordia (Fig. 2G,H) and were particularly high in rostral neocortical regions. Interestingly, expression of Ptch1 also followed a visible columnar pattern (Fig. $2 G, H$, arrows) along the anterior neocortex of the E12.5 Sufu-cKO mice. These findings indicated deregulation of SHH signaling in discrete neocortical progenitor subpopulations, and not differentiated neurons, in the E12.5 neocortex of Sufu-cKO mice.

\section{Altered molecular identity of progenitors in the E12.5 Sufu- cKO neocortex}

Since changes in SHH signaling activity in the neocortex are known to disrupt progenitor fate specification in late-stage corticogenesis (Komada et al., 2008a; L. Wang et al., 2016), we wondered whether the ectopic activation of SHH signaling at E12.5 initiated a cascade of disruptive differentiation events. Functional analysis of the transcriptome using the Database for Annotation, Visualization, and Integrated Discovery (DAVID; https://david.ncifcrf.gov/) (Huang et al., 2009) found overrepresentation of genes with gene ontology terms associated with neural development, commitment, specification, and differentiation (Fig. $3 A$ ). Further examination of specific gene sets showed relatively mild changes in the expression of genes typical of dorsal 
Table 3. Expression of genes associated with the SHH signaling pathway ${ }^{a}$

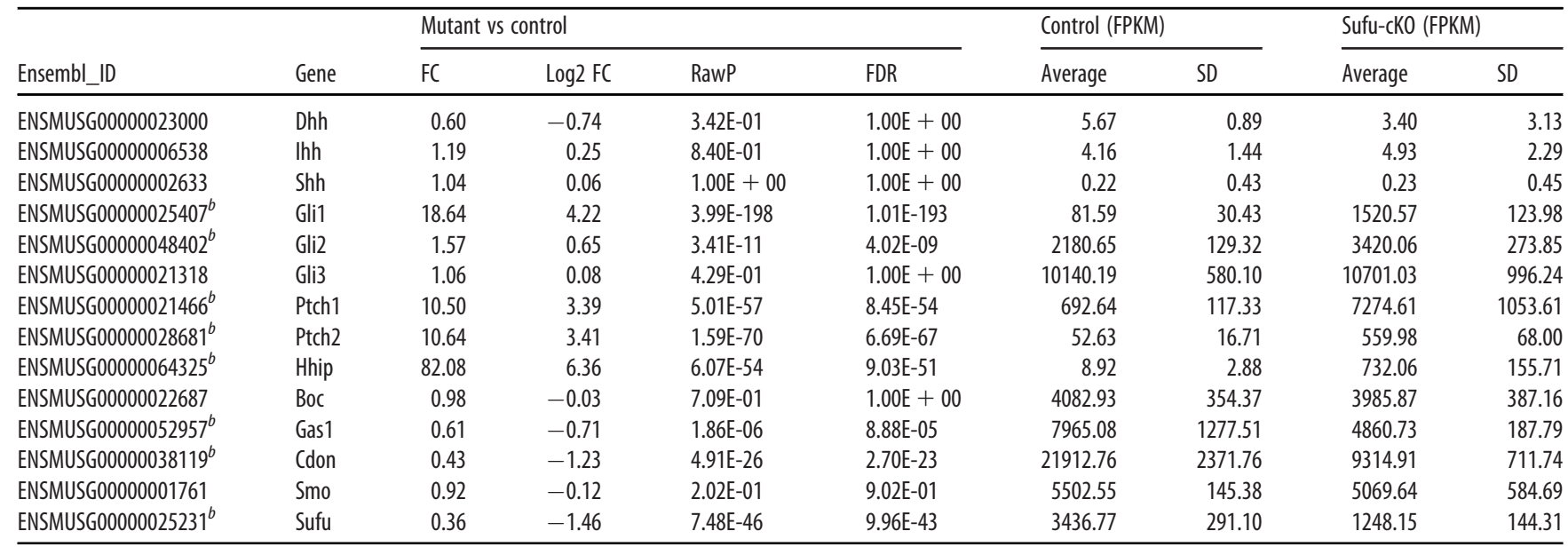

${ }^{a} \mathrm{FC}$, Unlogged fold change; Log2FC, log2 fold change; RawP, unadjusted $p$ value; FDR, $p$ value adjusted for multiple comparisons; average FPKM, average normalized sample values; SD, standard deviation of normalized sample values.

${ }^{b}$ Genes are significantly upregulated in the E12.5 Sufu-cKO dorsal forebrain ( $n=4$ mice per genotype).

forebrain progenitors (Fig. 3B; Table 4). Indeed, similar to Pax6 expression in Figure 1, other markers for dorsal forebrain cells, such as Tbr2, Lhx2, and Nr2f1, remained expressed, and may be even expressed at slightly higher levels in the mutant neocortex as observed with Pax6, Tbr1, Nr2f1, or Nr2f2 (Fig. $3 B$; Table 4). These findings validated the efficiency of the dissection and confirmed that the molecular identity of dorsal forebrain domains was established in the E12.5 Sufu-cKO neocortex.

Nevertheless, RNA levels for several ventral progenitor genes dramatically increased in the E12.5 Sufu-cKO neocortex compared with controls (Fig. 3B). We found a specific increase in the expression of subpallial-specific genes in the neocortex (Fig. 3B; Table 4). Moreover, while we previously did not observe a significant increase in Ascl1 protein expression in the E12.5 neocortex (Yabut et al., 2015), here we found significantly higher levels of Ascl1 transcript, despite not detecting Ascl1 protein (Extended Data Fig. 3-1A). Additionally, significant upregulation of genes normally expressed in the GE, such as Gsx2 and Dlx1/2 (Petryniak et al., 2007), was also ectopically expressed in the neocortex of E12.5 Sufu-cKO mice (Fig. 3B).

We subsequently conducted immunostaining or ISH experiments to validate the expression of subpallial-specific markers. In agreement with the transcript increase quantified by RNASeq, visibly higher levels of $\mathrm{Tbr} 2^{+}, \mathrm{NR} 2 \mathrm{~F} 1^{+}$, and $\mathrm{Lhx} 2^{+}$cells were observed across the anterior to posterior axis of the SufucKO neocortex compared with controls (Fig. 3B,C; Extended Data Fig. 3-1B,C). Similarly, ectopic expression of subpallial-specific genes Olig2, Dlx1, and Gsx1 was detected in the E12.5 SufucKO neocortex (Fig. 3D,E; Extended Data Fig. 3-1D). Expression of these genes was detected in the SVZ and VZ regions of the E12.5 Sufu-cKO neocortex and exhibited a columnar pattern, whereas these genes were absent in controls. Additionally, neocortical progenitors in these regions were improperly specified since we detected ectopic expression of the ventral forebrain progenitor marker, Olig2, in areas where Pax6 was absent in the E12.5 Sufu-cKO neocortex (Fig. 3F, arrows), whereas Olig2 was completely absent in the neocortex of control mice. This expression pattern persisted in the anterior regions of the E14.5 SufucKO neocortex but not in posterior regions (Extended Data Fig. 3-2A). However, we did not see similarly extensive disruptions in Tbr2 expression in the E12.5 Sufu-cKO neocortex, even in areas where Olig2 ${ }^{+}$cells were highly enriched (Fig. 3C,G). Nevertheless, while the majority of Tbr $2^{+}$cells in the E12.5 Sufu-cKO SVZ did not coexpress Olig2, a few cells within the VZ coexpressed Olig2 and Tbr2 (Fig. $3 G$, boxed inset, arrowheads). Further, by E14.5, Tbr2 ${ }^{+}$cells, similar to Pax6 ${ }^{+}$cells, became intermittent in the anterior neocortex of Sufu-cKO mice and were populated by Ascl1 ${ }^{+}$cells (Extended Data Fig. $3-2 B, C)$. Ectopic expression of Olig2 was not prevalent in the E11.5 Sufu-cKO neocortex, although we noted irregularities in Olig2 expression near the pallial-subpallial boundary (Extended Data Fig. 3-1E), indicating that a subset of aberrant progenitors may be present at this stage. Together, these findings establish that activation of $\mathrm{SHH}$ signaling in early stages of corticogenesis did not disrupt the regionalization of dorsoventral axis but has begun to destabilize the specification program of neocortical $\mathrm{RG}$ progenitors to disrupt the specification of Tbr $2^{+}$IPCs.

\section{Ectopic activation of SHH signaling upregulates FGF15 expression}

To determine how these genetic changes mediated specification defects in the E12.5 Sufu-cKO neocortex, we further analyzed the overall nature of differentially expressed genes from our RNA-Seq data (Table 2). Functional analysis of the transcriptome using DAVID showed enrichment of genes encoding proteins with roles in cell-cell communications, such as membrane-bound or extracellular matrix proteins in the E12.5 Sufu-cKO transcriptome (Fig. 4A; Table $5)$. Thus, the molecular makeup of the VZ/SVZ progenitor niche has been significantly altered in response to the ectopic activation of $\mathrm{SHH}$ signaling in the E12.5 Sufu-cKO neocortex. Among these is the gene encoding the secreted ligand, Fibroblast Growth factor 15 (Fgf15) (Fig. 4B). Fgf15 was a previously reported $\mathrm{SHH}$ signaling gene target in the developing cerebellum affecting neuronal precursor behavior (Gimeno and Martinez, 2007; Komada et al., 2008b; Kim et al., 2018). Similarly, we found that, in the E12.5 Sufu-cKO neocortex, Fgf15 dramatically increased (5.72 log2 fold change, $p<0.0001$ ), likely as a consequence of Gli3R loss. ISH using Fgf15 riboprobes confirmed these findings, with Fgf15 ectopically expressed throughout the neocortical wall of the E12.5 and E14.5 SufucKO mice while Fgf15 expression was relatively low in controls 
A

TOP GOTERM

\section{BIOLOGICAL PROCESSES}

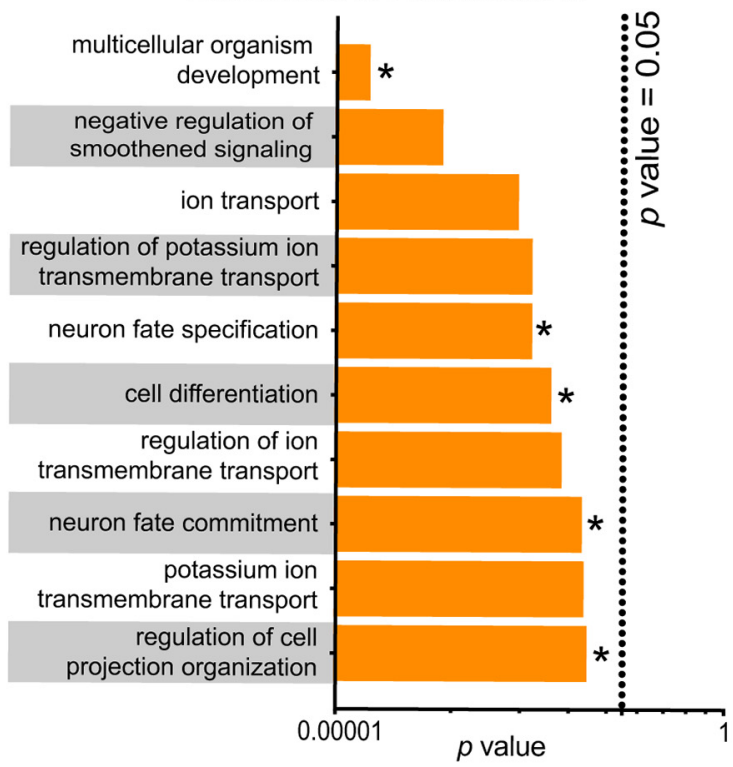

B

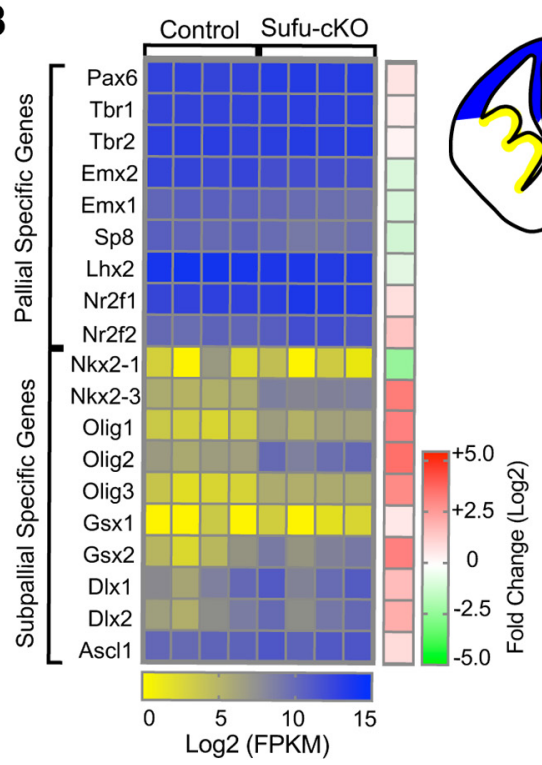

F
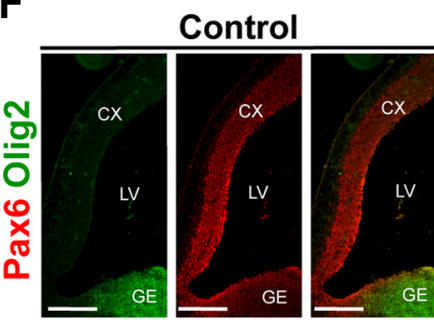

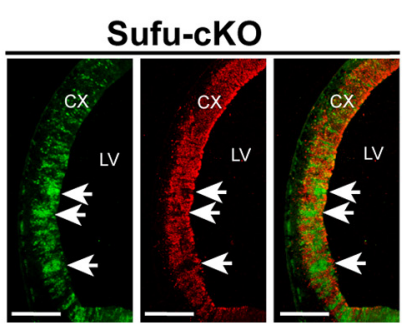

C
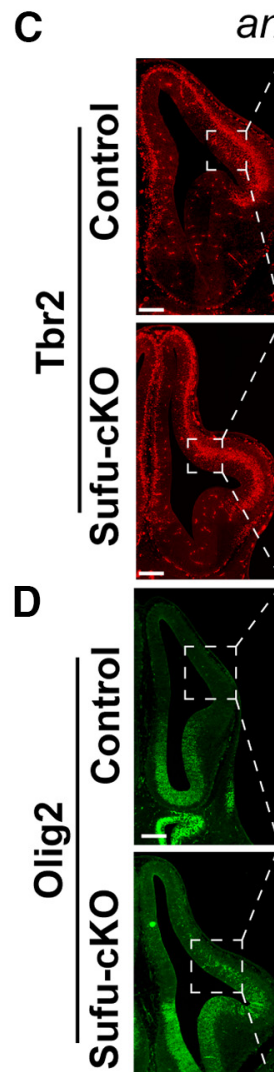

anterior

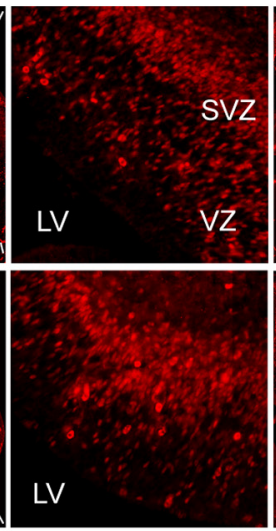

posterior

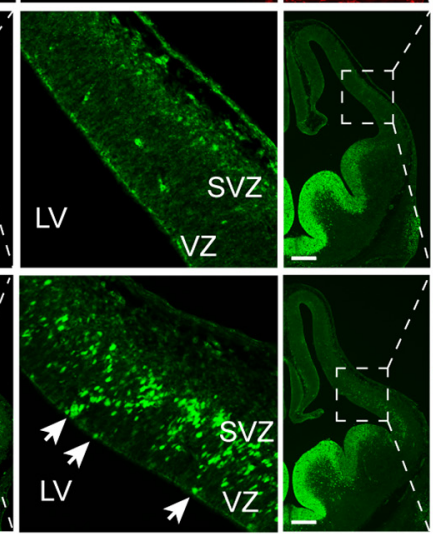

LV

SVZ

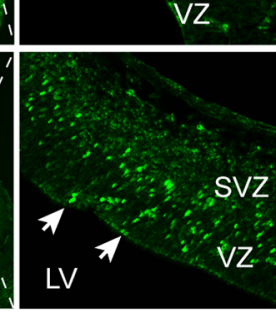

E

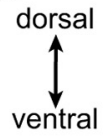

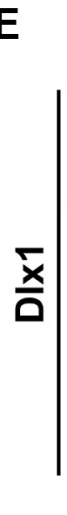

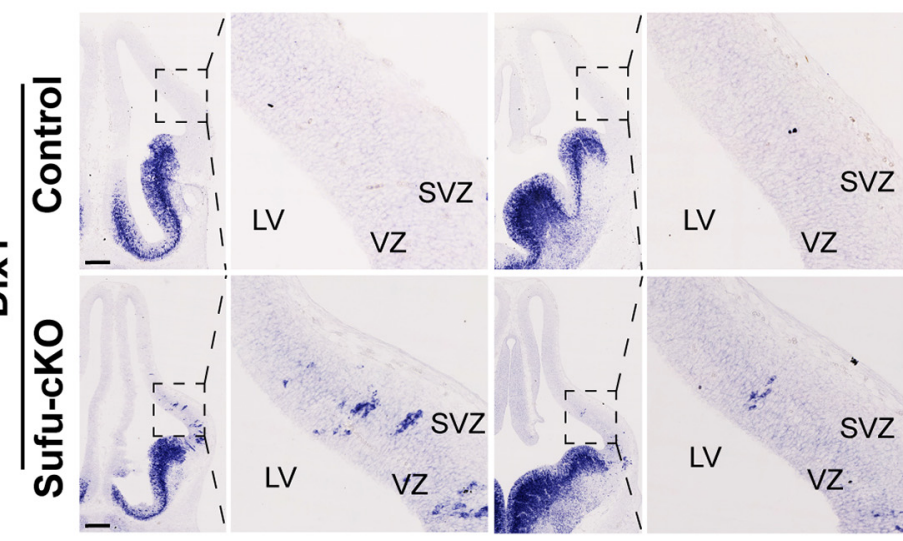

G
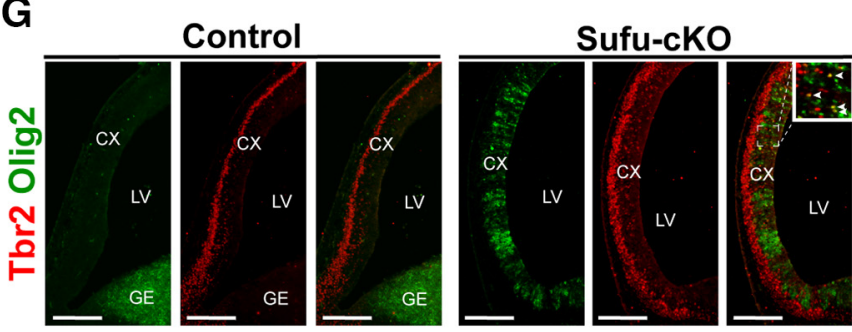

Figure 3. Increased expression of ventral progenitor markers in neocortical progenitors of E12.5 Sufu-cK0 embryos. $\boldsymbol{A}$, Functional annotation of differentially expressed genes identified by RNA-Seq show top GOTERM Biological Processes (with adjusted $p$ value $<0.05$ ) involve development, specification, differentiation, and fate commitment $(*)$. There is also a notable enrichment in ion transmembrane transport GOTERMs, reflecting disrupted electrophysiological properties because of abnormal differentiation of neurons or specific neuronal subtypes. $\boldsymbol{B}$, Heat map of select genes typically expressed by dorsal or ventral progenitors in individual control and Sufu-cKO mice ( $n=4$ mice per genotype). RNA levels (Log2 FPKM scale) reflect mild differences in expression of dorsal progenitor genes (reflected by fold change scale), while dramatic differences in expression levels of ventral progenitor genes are observed between controls and Sufu-cKO dorsal forebrain (see also Table 4). C, Immunofluorescence staining for pallial-specific marker, Tbr2, in coronal sections of the E12.5 Sufu-cKO and control forebrain showed exclusive expression in the neocortex across the A-P axis. Pallial-specific markers NR2F1 and Lhx2 were similarly confined in the dorsal forebrain (Extended Data Fig. 3-1B,C). Scale bar, 100 $\mu$ m. $\boldsymbol{D}, \boldsymbol{E}$, Immunofluorescence staining for Olig2 (D) and ISH for DIx1 $(\boldsymbol{E})$ on coronal sections of the E12.5 control and Sufu-cKO forebrain validate the ectopic expression of subpallial-specific genes across the A-P axis of the E12.5 Sufu-cKO neocortex, whereas these genes were absent in controls. Olig2- and Dlx1- expressing cells largely localized in the VZ and SVZ. Some groups of cells expressing 
Table 4. Expression of pallial- and subpallial-specific genes ${ }^{a}$

\begin{tabular}{|c|c|c|c|c|c|c|c|c|c|c|}
\hline & \multirow[b]{2}{*}{ Ensembl_ID } & \multirow[b]{2}{*}{ Gene } & \multicolumn{4}{|c|}{ Mutant vs control } & \multicolumn{2}{|l|}{ Controls (FPKM) } & \multicolumn{2}{|l|}{ Sufu-cKO (FPKM) } \\
\hline & & & $\mathrm{FC}$ & $\log 2 \mathrm{FC}$ & RawP & FDR & Average & SD & Average & SD \\
\hline \multirow{6}{*}{ Pallial-specific genes } & ENSMUSG00000027168 & Pax6 & 1.45178268 & 0.53782551 & $1.004 \mathrm{E}-08$ & 7.6972E-07 & 7334.1262 & 557.776073 & 10647.5574 & 579.137216 \\
\hline & ENSMUSG00000032446 & Eomes & 1.09461657 & 0.1304256 & 0.15380485 & 0.79883164 & 8778.80064 & 946.263097 & 9609.42061 & 765.318 \\
\hline & ENSMUSG00000043969 & Emx2 & 0.60690796 & -0.7204503 & 1.1807E-13 & $2.0184 \mathrm{E}-11$ & 6169.69045 & 604.010197 & 3744.43426 & 268.720823 \\
\hline & ENSMUSG00000033726 & Emx1 & 0.60444715 & -0.7263119 & $3.255 \mathrm{E}-06$ & 0.00014653 & 1588.14413 & 187.604638 & 959.9492 & 200.078024 \\
\hline & ENSMUSG00000069171 & $\mathrm{Nr} 2 \mathrm{f1}$ & 1.59051974 & 0.66949828 & 0.00014645 & 0.00415822 & 7171.74849 & 888.316712 & 11406.8075 & 2022.77936 \\
\hline & ENSMUSG00000030551 & $\mathrm{Nr} 2 \mathrm{f} 2$ & 2.62720361 & 1.39352802 & 0.00048355 & 0.01154083 & 1231.1201 & 229.798831 & 3234.40319 & 1174.67098 \\
\hline \multirow[t]{6}{*}{ Subpallial-specific genes } & ENSMUSG00000041911 & Dlx1 & 3.09901298 & 1.6318088 & 0.07970986 & 0.56981627 & 461.094387 & 506.237194 & 1428.93749 & 954.933235 \\
\hline & ENSMUSG00000023391 & Dlx2 & 3.95059159 & 1.98206871 & 0.02352951 & 0.25117012 & 184.932474 & 200.493153 & 730.592677 & 453.539091 \\
\hline & ENSMUSG00000020052 & Ascl1 & 1.72359337 & 0.78541945 & 0.00107784 & 0.02236943 & 1331.20054 & 187.629663 & 2294.44843 & 561.105261 \\
\hline & ENSMUSG00000053129 & Gsx1 & 1.35616481 & 0.43953252 & 0.85642916 & 1 & 2.18572234 & 4.37144469 & 2.96419973 & 2.64163128 \\
\hline & ENSMUSG00000001496 & Nkx2-1 & 0.22177988 & -2.1727996 & 0.39910601 & 1 & 25.2811711 & 45.3686169 & 5.6068551 & 6.573452 \\
\hline & ENSMUSG00000044220 & Nkx2-3 & 10.1453116 & 3.34274127 & 7.382E-20 & $2.1222 \mathrm{E}-17$ & 34.6890788 & 7.47294372 & 351.931514 & 86.7310817 \\
\hline
\end{tabular}

${ }^{a}$ Expression of select genes typically expressed by dorsal or ventral progenitor in individual control and Sufu-cK0 mice ( $n=4$ mice per genotype). FC, Unlogged fold change; Log2FC, log2 fold change; RawP, unadjusted $p$ value; FDR, $p$ value adjusted for multiple comparisons; average FPKM, average normalized sample values; SD, standard deviation of normalized sample values.

(Fig. 4C,D). We also observed upregulation of Fgf15 in embryos in which Smo was constitutively active in neocortical progenitors (Emx1-Cre;SmoM2 or SmoM2-cA) (Long et al., 2001), confirming the role of activated SHH signaling in inducing Fgf15 gene expression in the embryonic neocortex (Extended Data Fig. 4-1). Importantly, Fgf15 expression in the E12.5 Sufu-cKO was detected along the VZ/SVZ, and particularly overlapped with Ptch1-expressing cells in the VZ (Fig. 4E). These observations indicated that ectopic Fgf15 expression was induced in $\mathrm{RG}$ progenitors along the $\mathrm{VZ}$ and persisted in IPCs as a consequence of loss of Sufu and deregulated $\mathrm{SHH}$ signaling.

\section{Upregulated FGF15 expression correlates with ectopic activation of MAPK signaling in neocortical progenitor zones}

FGF15 preferentially binds to its cognate receptor, FGF receptor 4 (FGFR4), to activate intracellular signaling cascades, particularly the Ras/mitogen-activated protein kinase (MAPK) pathway

$\leftarrow$

Olig2 and D|x1 also appeared in columnar arrangement (arrows). Similar columnar pattern was also detected in cells ectopically expressing another subpallial-specific marker, Gsx2 (Extended Data Fig. 3-1D). Few Olig2 ${ }^{+}$cells also began to exhibit an irregular pattern along the pallial-subpallial boundary in the E11.5 Sufu-cKO forebrain (Extended Data Fig. 3-1E). Scale bar, $100 \mu \mathrm{m}$. $\boldsymbol{F}$, Double immunofluorescence staining on E12.5 sagittal sections with Pax6 and 0lig2 showed ectopic expression of 0lig2 in areas where Pax6 is missing in the Sufu-cK0 neocortex (arrows), whereas Olig2 was not expressed in this region in the control neocortex. However, Ascl1 was not similarly affected since protein levels of Ascl1 were low in the E12.5 Sufu-cKO and control neocortex (Extended Data Fig. 3-1A). This pattern of Olig2 and Pax6 expression continued in the E14.5 neocortex, when ectopic expression of Ascl1 was also prevalent in the VZ/SVZ (Extended Data Fig. 3-2A,B). Scale bar, $250 \mu \mathrm{m}$. G, Double immunofluorescence staining with Tbr2 and Olig2 on sagittal sections of E12 Sufu-CKO and control littermates showed that, unlike $\mathrm{Pax}^{+}$cells, the distribution of $\mathrm{Tbr}^{+}$cells was not affected in the anterior regions where ectopic expression of Olig2 was present. However, $\mathrm{Tbr}^{+}$cells were found to coexpress Olig2 in more anterior regions of the E12.5 Sufu-cKO neocortex. By E14.5, areas where $\mathrm{Tbr}^{+}$cells were absent in the anterior neocortex, showed Ascl1expressing cells (Extended Data Fig. 3-2C). Scale bar, $250 \mu \mathrm{m}$.
(Guillemot and Zimmer, 2011). Indeed, in the neocortex of E12.5 Sufu-cKO mice, MAPK signaling pathway activity, as marked by phosphorylated-ERK1/2 (pERK1/2 ${ }^{+}$) labeling, is visibly upregulated unlike controls (Fig. 5A). We found that pERK1/ $2^{+}$areas occupied the progenitor-rich VZ/SVZ neocortical regions, whereas it was largely undetected in similar neocortical regions in controls. Quantification of $\mathrm{pERK} 1 / 2^{+}$regions in representative sections across the dorsal forebrain (Fig. 5 C) showed a consistently larger area with pERK $1 / 2^{+}$immunoreactivity in the E12.5 Sufu-cKO cortex compared with controls (two-way ANOVA, $p=0.0365, n=5 \mathrm{control} / \mathrm{Sufu}-\mathrm{cKO}$ embryos) (Fig. $5 D$; Table 1). This remained true at E14.5, where pERK $1 / 2^{+}$-rich regions were detected further toward the dorsal regions of the Sufu-cKO neocortex (Fig. 5B). Quantification of pERK1/2 regions in the E14.5 neocortex confirmed these observations and showed a significant increase in Sufu-cKO mice (two-way ANOVA, $p=0.013, n=3-5$ control/Sufu-cKO embryos) (Fig. $5 E$; Table 1). At both E12.5 and E14.5 time points, cells labeled with pERK $1 / 2^{+}$clearly overlapped with FGF15-expressing VZ/SVZ areas in the Sufu-cKO neocortex (Fig. 4D). Together, these observations indicated that loss of Sufu resulted in the overexpression of FGF15 in the neocortex, subsequently driving the ectopic activation of FGF signaling to activate intracellular MAPK signaling in neocortical progenitors.

\section{FGF15 upregulation is required to elicit SHH signaling-} mediated defects in the production and specification of IPCs Reduction in IPCs is a consistent phenotype in the embryonic neocortex of mice with excessive levels of SHH signaling, including Sufu-cKO mice (Komada et al., 2008a; Dave et al., 2011; Yabut et al., 2015). We therefore investigated whether downregulation of IPCs in the neocortex because of ectopic SHH signaling is directly mediated by FGF15 signaling. To test this, we cultured WT forebrain slices from the anterior regions of E12.5 control and Sufu-cKO embryos (Fig. 6A). Forebrain organotypic cultures maintain the 3D structure of the VZ/SVZ niche, allowing for careful examination of how precisely added compounds affect progenitor behavior over time. Forebrain slices cultured for 

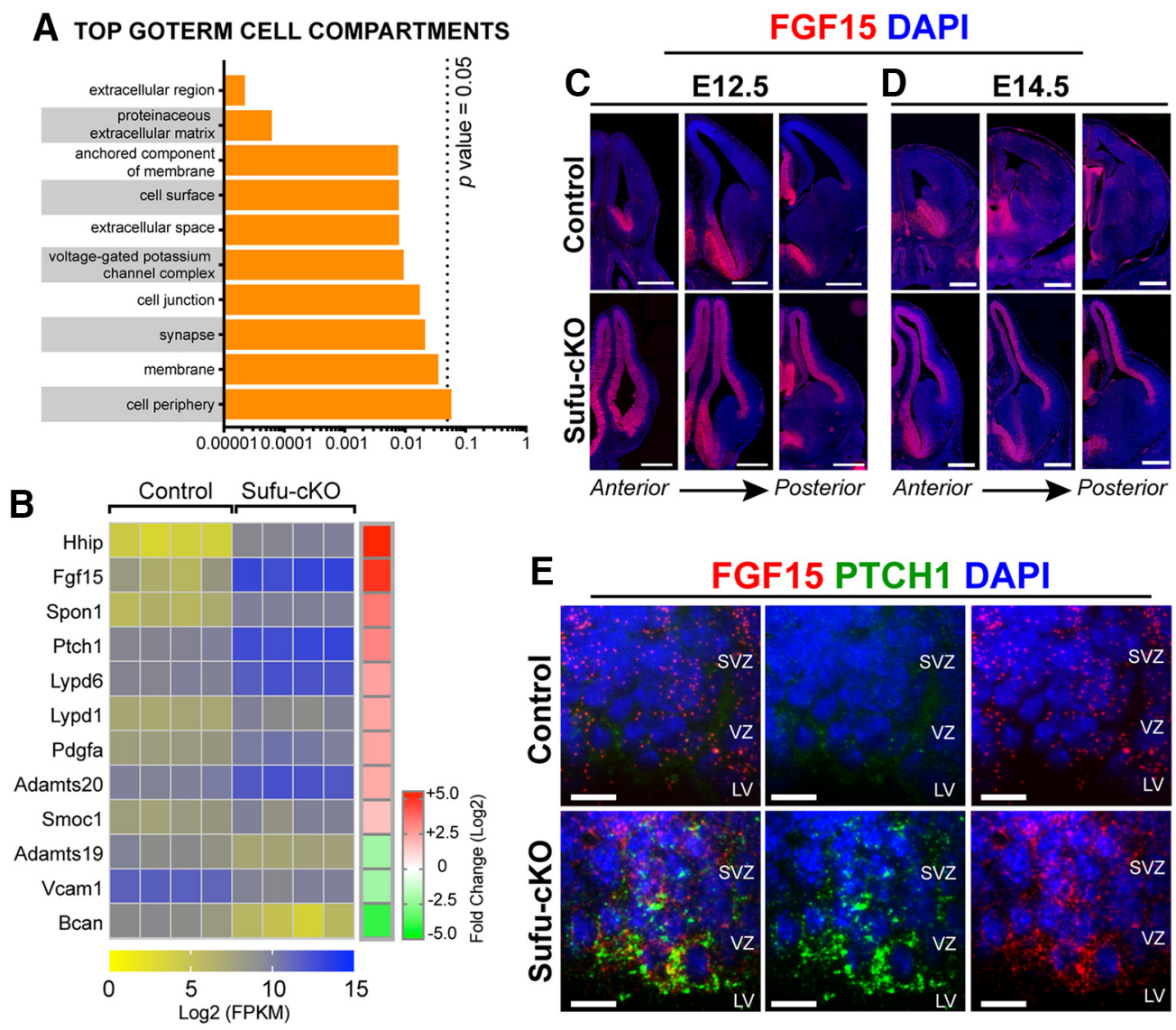

Figure 4. Ectopic activation of SHH signaling drives Fgf15 expression in neocortical progenitors of E12.5 Sufu-cKO embryos. A, Functional annotation of differentially expressed genes identified by RNA-Seq showing the majority of genes encode proteins that localize to extracellular matrix or cell surface/membrane as the top GOTERMs cell compartments (with adjusted $p$ value $<0.05$ ). $\boldsymbol{B}$, Heat map of top differentially expressed genes encoding extracellular matrix or cell membrane-bound proteins between control and Sufu-cK0 mice ( $n=4$ mice per genotype). RNA levels (Log2 FPKM scale) show expression of Fgf15 is significantly upregulated (reflected by fold change scale in the E12.5 Sufu-cKO dorsal forebrain; see also Table 5). C, D, ISH for Fgf15 in the E12.5 and E14.5 control and Sufu-cKO neocortex. High levels of Fgf15 expression were detected throughout the E12.5 Sufu-cKO dorsal forebrain, and particularly enriched in the VZ/SVZ regions, whereas Fgf15 expression was detected only in ventral forebrain regions in controls (C). Expression of Fgf15 persisted in the E14.5 control and Sufu-cKO forebrains (D). Ectopic expression of Fgf15 was also detected in transgenic mice carrying constitutively active Smoothened allele (Extended Data Fig. 4-1). These findings confirmed that activation of SHH signaling and loss of Sufu force Fgf15 expression in the embryonic neocortex. Scale bar, $500 \mu \mathrm{m}$. $\boldsymbol{E}$, Multiplex RNAscope ISH of Ptch1 and Fgf15 riboprobes on E12.5 brains did not detect Ptch1 expression, whereas low levels of Fgf15 expression were detected in the VZ and SVZ of the neocortex of controls. In the E12.5 Sufu-cK0 neocortex, high levels of Ptch1 and Fgf15 colocalization were detected in the VZ and SVZ. Sections are counterstained with DAPI. Scale bar, $10 \mu \mathrm{m}$.

Table 5. List of genes encoding extracellular matrix or cell membrane-bound proteins ${ }^{a}$

\begin{tabular}{|c|c|c|c|c|c|c|c|c|c|}
\hline \multirow[b]{2}{*}{ Gene } & \multirow[b]{2}{*}{ Description } & \multicolumn{4}{|c|}{ Mutant vs control } & \multicolumn{2}{|l|}{ Controls (FPKM) } & \multicolumn{2}{|c|}{ Sufu-cKO (FPKM) } \\
\hline & & $\mathrm{FC}$ & $\log 2 \mathrm{FC}$ & RawP & FDR & Average & SD & Average & SD \\
\hline Hhip & Hedgehog-interacting protein & 82.0823811 & 6.35900068 & $6.07 \mathrm{E}-54$ & $9.03 \mathrm{E}-51$ & 8.91857894 & 2.87906586 & 732.058195 & 155.710915 \\
\hline Fgf15 & FGF15 & 52.7038764 & 5.71983717 & $1.38 \mathrm{E}-96$ & $1.16 \mathrm{E}-92$ & 152.277335 & 111.900656 & 8025.60586 & 1237.95433 \\
\hline Spon1 & spondin 1, (f-spondin) extracellular matrix protein & 14.9532123 & 3.90238353 & $2.55 \mathrm{E}-158$ & $3.23 \mathrm{E}-154$ & 56.2092056 & 22.9496826 & 840.508183 & 67.3977619 \\
\hline Ptch1 & patched homolog 1 & 10.5027097 & 3.39268968 & $5.01 E-57$ & $8.45 E-54$ & 692.640882 & 117.331353 & 7274.60609 & 1053.60989 \\
\hline Lypd6 & LY6/PLAUR domain containing 6 & 6.23322426 & 2.63997862 & $3.73 \mathrm{E}-23$ & $1.55 \mathrm{E}-20$ & 714.738273 & 124.510077 & 4455.12394 & 879.153524 \\
\hline Lypd1 & Ly6/Plaur domain containing 1 & 5.78045668 & 2.53118348 & $1.68 \mathrm{E}-14$ & $3.08 \mathrm{E}-12$ & 108.297771 & 11.4653093 & 626.010575 & 155.439255 \\
\hline Pdgfa & platelet derived growth factor, alpha & 5.60936415 & 2.48783724 & $8.41 \mathrm{E}-17$ & $1.95 \mathrm{E}-14$ & 213.357738 & 41.0886507 & 1196.80125 & 267.580448 \\
\hline Adamts20 & $\begin{array}{l}\text { a disintegrin-like and metallopeptidase (reprolysin type) with thrombospondin } \\
\text { Type } 1 \text { motif, } 20\end{array}$ & 5.09486349 & 2.34904349 & $8.48 \mathrm{E}-35$ & $7.66 \mathrm{E}-32$ & 879.123707 & 153.586556 & 4479.01527 & 645.467259 \\
\hline Smoc1 & SPARC related modular calcium binding 1 & 3.28121386 & 1.71422963 & 5.24E-06 & 0.00022471 & 195.520108 & 59.5128888 & 641.543288 & 199.481655 \\
\hline Adamts19 & $\begin{array}{l}\text { a disintegrin-like and metallopeptidase (reprolysin type) with thrombospondin } \\
\text { Type } 1 \text { motif, } 19\end{array}$ & 0.25950133 & -1.9461861 & $3.80 \mathrm{E}-07$ & $2.12 \mathrm{E}-05$ & 505.608463 & 158.402559 & 131.20607 & 17.3208447 \\
\hline Vcam1 & vascular cell adhesion molecule 1 & 0.25534623 & -1.9694734 & $8.06 \mathrm{E}-61$ & $2.04 E-57$ & 2919.72302 & 248.427664 & 745.540259 & 144.812934 \\
\hline Bcan & brevican & 0.06566811 & -3.9286633 & $2.64 \mathrm{E}-13$ & $4.31 \mathrm{E}-11$ & 438.508098 & 149.646904 & 28.795998 & 17.7145662 \\
\hline
\end{tabular}

${ }^{a}$ Top differentially expressed genes encoding extracellular matrix or cell membrane-bound proteins between the E12.5 control and Sufu-cKO dorsal forebrain ( $n=4$ embryos per genotype). $\mathrm{FC}$, Unlogged fold change; Log2FC, log2 fold change; RawP, unadjusted $p$ value; FDR, $p$ value adjusted for multiple comparisons; average FPKM, average normalized sample values; SD, standard deviation of normalized sample values. 


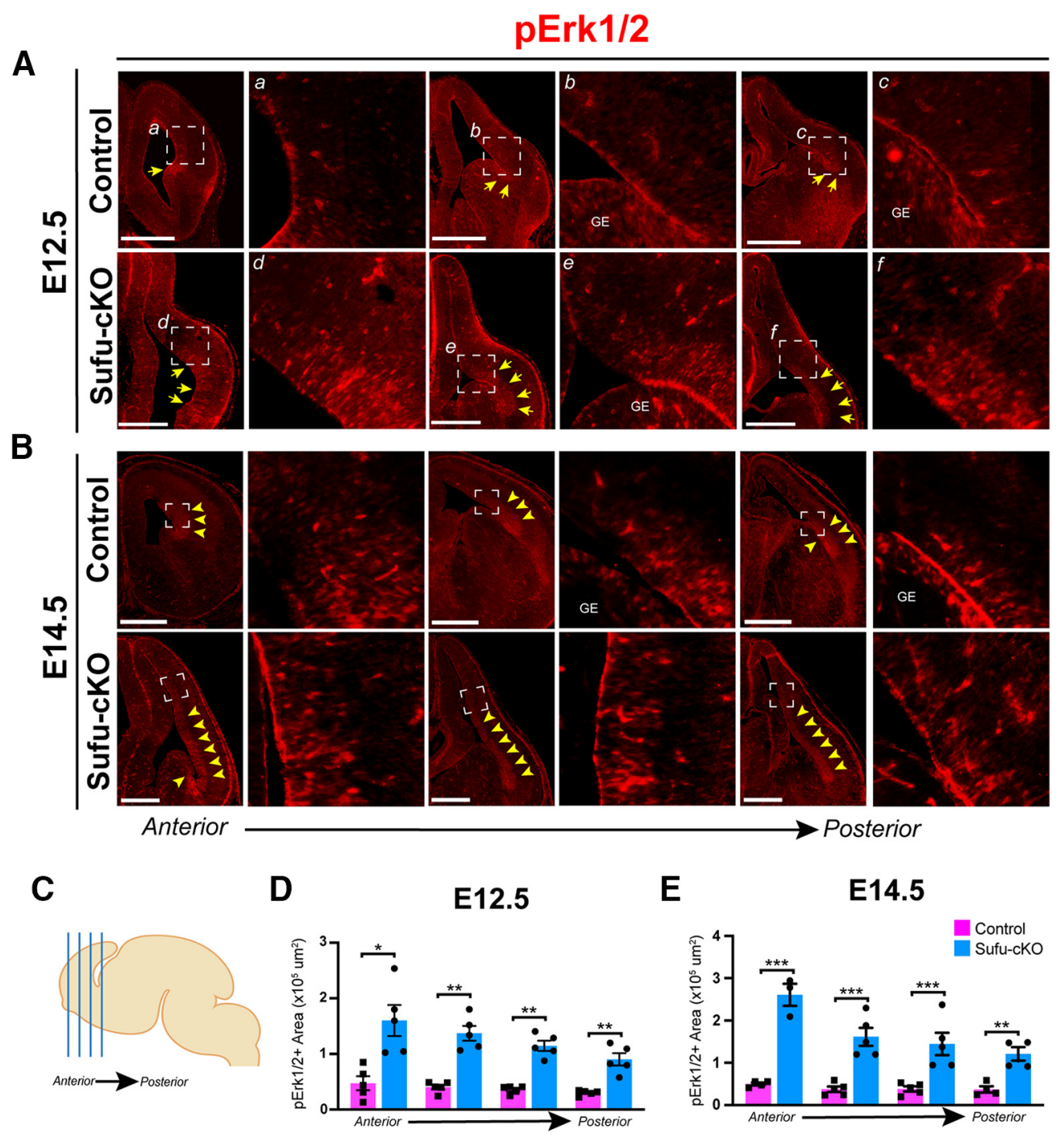

Figure 5. Upregulated FGF15 expression correlates with ectopic activation of MAPK signaling in neocortical progenitor zones. $\boldsymbol{A}, \boldsymbol{B}$, Immunofluorescence staining against phosphorylated Erk1/2 (pErk1/2) was conducted on E12.5 and E14.5 control and Sufu-CKO brains to detect for MAPK signaling activation. pErk1/2-expressing cells (pErk1/2 ${ }^{+}$) were detected along the pallialsubpallial boundary (PSB) of the E12.5 control and Sufu-cKO forebrain (white arrows). However, pErk1/2 ${ }^{+}$cells were found from the PSB and the lateral cortex, particularly in the VZ/SVZ regions (yellow arrows, $\boldsymbol{A}$ ). At E14.5, pErk1/2 ${ }^{+}$cells expanded dorsally within the VZ/SVZ regions in both control and Sufu-cK0 neocortex $(B)$. However, pErk1/2 ${ }^{+}$cells in the Sufu-cK0 neocortex greatly expanded compared with controls (yellow arrows). Scale bars: $500 \mu \mathrm{m}(\boldsymbol{A}, \boldsymbol{B})$. $\mathbf{C}-\boldsymbol{E}$, Four representative sections across the A-P axis of the forebrain $(\boldsymbol{C})$ were sampled to measure pERK1/2 ${ }^{+}$regions in the E12.5 and E14.5 control and Sufu-CKO mice. Bar graphs of quantification of pErk1/2 ${ }^{+}$regions in the neocortex of E12.5 (D) and E14.5 (E) control and Sufu-cK0 mice ( $n=3-5$ embryos per genotype) represent significant interaction between position and genotype (repeated-measures two-way ANOVA, $p=0.0365$ ). Significance between genotypes in PhErk1/2 ${ }^{+}$regions in the Sufu-CKO neocortex at both E12.5 and E14.5, particularly in anterior regions: ${ }^{*} p \leq 0.05$; ${ }^{* *} p \leq 0.01$; ${ }^{* * *} p \leq 0.001$; repeated-measures, two-way ANOVA with HolmSidak's multiple comparisons test. Values of statistics are shown in Table 1. Bar graphs represent average values. Error bars indicate SEM.

2 DIV maintain their anatomic features with well-preserved dorsal and ventral domains. Neocortical IPCs typically expressing Tbr2 (Hevner, 2019) were exclusively observed in the dorsal forebrain whereas ventral forebrain progenitors were typically expressing Olig2 (Miyoshi et al., 2007) (Fig. 6B). Addition of various compounds altered IPC numbers in neocortical regions of forebrain slices (one-way ANOVA, $p=0.0001, n=3$ per treatment condition) (Fig. $6 C, D)$. SHH ligands significantly decreased the number of $\mathrm{Tbr} 2{ }^{+}$cells in neocortical slices after 2 DIV compared with mocktreated controls (SHH-treated $=3792 \pm 913.9$ cells $/ \mathrm{mm}^{2}$; DMSOtreated $=9207 \pm 303.5$ cells $/ \mathrm{mm}^{2}$; Holm-Sidak's multiple comparisons test, $p=0.0004)$. Similarly, Tbr2 ${ }^{+}$IPCs were significantly reduced on addition of FGF15 alone (FGF15-treated $=6448 \pm 526.8$ cells $/ \mathrm{mm}^{2}$; Holm-Sidak's multiple comparisons test, $\left.p=0.05\right)$ or with SHH (FGF15 + SHH-treated $=4511 \pm 645.7$ cells $/ \mathrm{mm}^{2}$; HolmSidak's multiple comparisons test, $p=0.0014$ ). However, addition of cyclopamine, which inhibits SHH signaling by rendering Smo inactive, did not alter the number of $\mathrm{Tbr}^{+}$IPCs (cyclopaminetreated $=8736 \pm 644$ cells $/ \mathrm{mm}^{2}$; Holm-Sidak's multiple comparisons test, $p=0.6585$ ). Instead, addition of cyclopamine and FGF15 significantly reduced the number of IPCs after 2 DIV (cyclopamine + FGF15-treated $=5550 \pm 187.3$ cells $/ \mathrm{mm}^{2} ;$ Holm-Sidak's multiple comparisons test, $p=0.0095)$. These findings indicated that blocking transmembrane proteins upstream of the $\mathrm{SHH}$ signaling pathway cannot sufficiently alter IPC production. Additionally, expression of downstream SHH gene targets, particularly FGF15, is required to exert changes in neocortical IPCs of the developing neocortex.

High levels of FGF15 alter the specification program of neocortical progenitors

Ectopic SHH signaling in the developing neocortex ultimately results in the production of confused progenitors unable to 
A

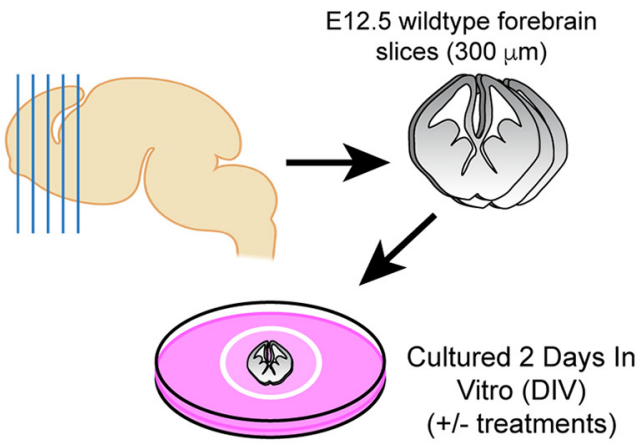

C

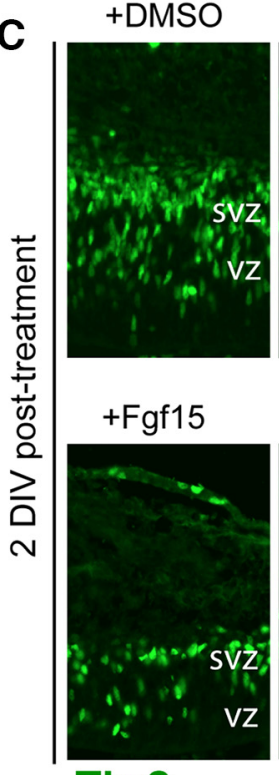

Tbr2

+ Fgf15

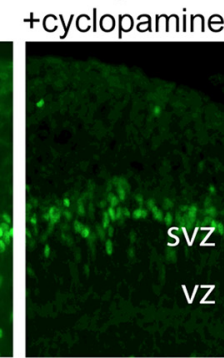

SVZ
VZ

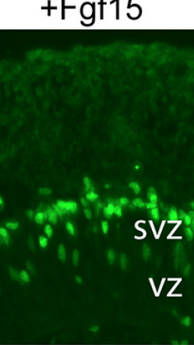

B Organotypic slice culture after 2 DIV Tbr2 Olig2 DAPI

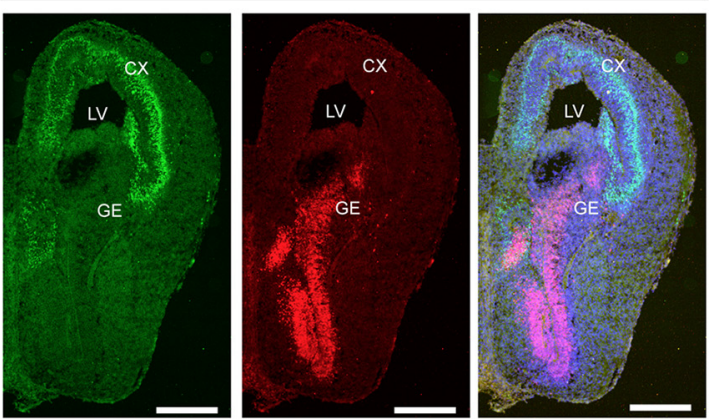

D
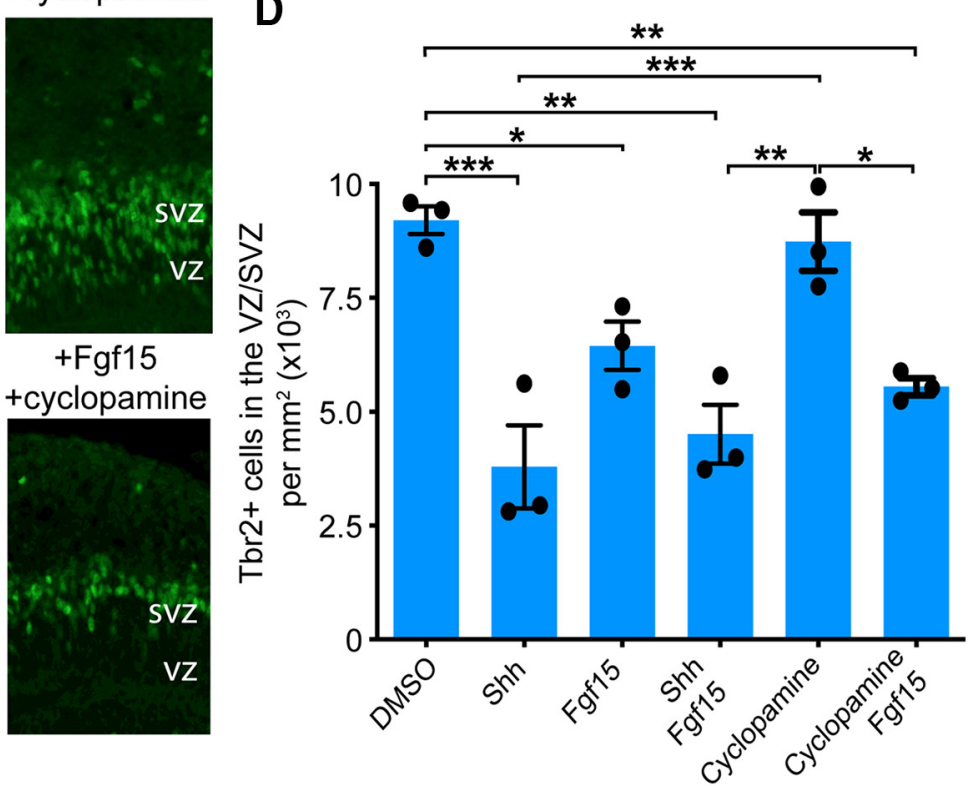

Figure 6. SHH signaling activates FGF15 signaling to inhibit production of neocortical IPCs. A, Diagram of experimental design for organotypic forebrain slice cultures from WT E12.5 brains. B, Immunofluorescence staining for dorsal (Tbr2, green) and ventral (0lig2, red) forebrain markers show exclusive localization of Tbr2-expressing and 0lig2-expressing cells in dorsal and ventral forebrain regions, respectively. Merged images represent no overlap in Tbr2 or 0lig2 labeling. Scale bar, $500 \mu \mathrm{m}$. C, Immunofluorescence staining with Tbr2 of sectioned organotypic slice cultures fixed after 2 DIV. Slices treated with $200 \mathrm{ng} / \mathrm{ml} \mathrm{SHH}$ and $100 \mathrm{ng} / \mathrm{ml} \mathrm{FGF15} \mathrm{show} \mathrm{reduced} \mathrm{numbers} \mathrm{of} \mathrm{Tbr2}{ }^{+}$IPCs compared with slices treated with DMSO or $5 \mu \mathrm{m}$ cyclopamine. Combined FGF15 and SHH or FGF15 and cyclopamine also show reduced Tbr2 ${ }^{+}$IPCs. Quantification of Tbr2 ${ }^{+}$cells per unit area (D) confirm significant interaction between treatments (repeated-measures two-way ANOVA, $p=0.0001$ ). Significant differences in Tbr2 ${ }^{+}$IPCs in SHH and FGF15-treated slice cultures ( $n=3$ experiments [2 or 3 slices each experiment] per treatment condition). Significance between treatment conditions: ${ }^{*} p \leq 0.05 ;{ }^{* *} p \leq 0.01 ;{ }^{* * *} p \leq 0.001$; repeated-measures, one-way ANOVA with Holm-Sidak's multiple comparisons test. Values of statistics are shown in Table 1. Bar graphs represent average values. Error bars indicate SEM.

maintain a specified neocortical neural fate (Yabut et al., 2015). The expansive ectopic activation of MAPK signaling, capable of altering neocortical progenitor fate (Y. Wang et al., 2012), in the Sufu-cKO embryonic neocortex is a likely consequence of increasing levels of FGF15. We tested this by adding FGF15 in organotypic forebrain cultures and examined whether this alone altered the fate of neocortical progenitors based on Olig2 expression. Indeed, we found that, after 2 DIV, the decrease in Tbr2 ${ }^{+}$ IPCs correlated with an obvious increase in Olig2 ${ }^{+}$cells in FGF15-treated slices compared with DMSO-treated controls (Fig. 7A-D). Further, low levels of Olig2 expression were detected in the VZ region, where Olig2 is typically not expressed in controls. Rather, in FGF15-treated slices, Olig2 ${ }^{+}$cells may coexpress low levels of Tbr2, indicating that treatment of FGF15 began to alter the identity of RG progenitors transitioning into IPCs (Fig. $7 \mathrm{G}, \mathrm{H}$, arrows). Indeed, many Olig2 ${ }^{+}$cells in the SVZ also expressed Tbr2 in FGF15-treated slices compared with DMSOtreated controls (Fig. 7E,F). Our quantification confirmed these observations, showing the $\sim 4.5$-fold increase in misspecified $\mathrm{Tbr}^{+}$cortical progenitors in FGF15-treated slices compared with DMSO-treated controls (FGF15-treated $=18.2 \pm 4.8$ cells $/$ $\mathrm{mm}^{2}$; DMSO-treated $=4.03 \pm 1.6$ cells $/ \mathrm{mm}^{2}$; unpaired $t$ test, $p=0.0186$ ) (Fig. 7I). These findings indicate that excessive levels of FGF15 can sufficiently alter the identity of neocortical progenitors, leading to the failure to maintain a proper specification program in the developing neocortex.

\section{Discussion}

Excitatory neurons in the mammalian neocortex are generated in a limited period at embryonic stages and mature into molecularly diverse subpopulations at postnatal stages. A strict 
+DMSO
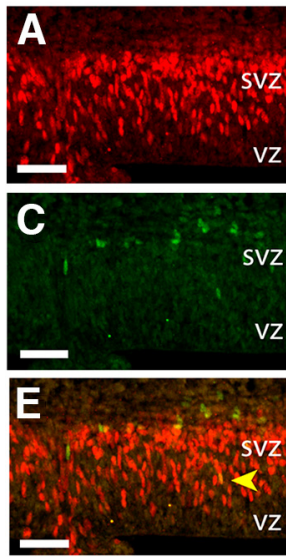

Olig2 Tbr2
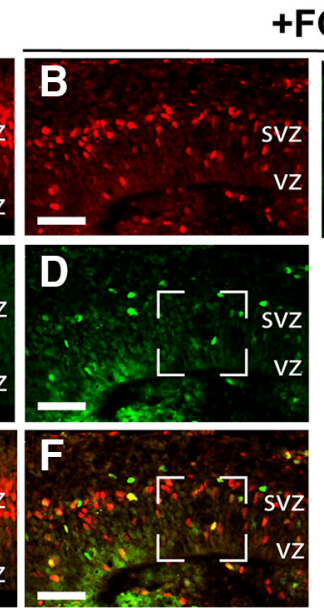

$\therefore$ atises
C
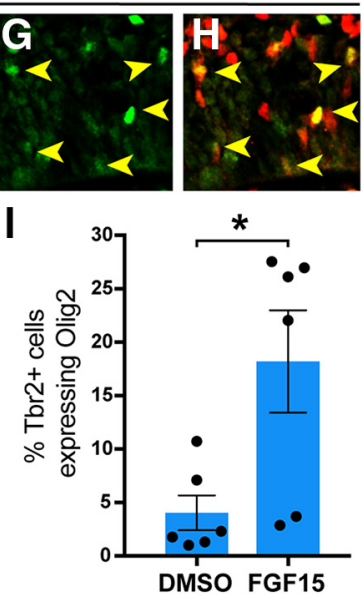

Figure 7. Increasing FGF15 levels alter the specification program of neocortical progenitors. $\boldsymbol{A}-\boldsymbol{F}$, Double immunofluorescence staining with IPC marker Tbr2 (red) and the ventral progenitor marker 0lig2 (green) on organotypic slice cultures fixed 2 DIV and after treatment. DMSO-treated slices show an abundance of Tbr2 ${ }^{+}$IPCs in the SVZ (A) and some 0lig2-expressing cells outside of the VZ/SVZ area $(\boldsymbol{B})$. In contrast, Tbr2 ${ }^{+}$IPCs in FGF15-treated slices are fewer $(\boldsymbol{B})$ and more 0 lig2 ${ }^{+}$cells are present in the VZ/SVZ $(\boldsymbol{F})$. Although Tbr2 ${ }^{+}$cells expressing 0 lig2 were also sometimes observed in DMS0-treated slices (yellow arrow, $\boldsymbol{C}$ ), the amount of double-labeled in FGF15-treated slices were visibly higher in the VZ/SVZ (G,H, yellow arrows). Scale bar, $50 \mu \mathrm{m}$. I, Graph represents the $\%$ of Tbr $2^{+}$cells colabeled with Olig2 in the VZ/SVZ of DMSO- and FGF15-treated slices ( $n=3$ experiments 2 or 3 slices each experiment] per treatment condition). The percentage of $\mathrm{Tbr}^{+}$coexpressing 0 lig2 is approximately fourfold in FGF15treated over control DMSO-treated slices and is significantly higher $\left({ }^{*} p \leq 0.05\right)$. Values of statistics are shown in Table 1. Bar graphs represent average values. Error bars indicate SEM.

specification program is maintained by neural progenitors to generate precise numbers and lineages, relying on spatially and temporally modulated molecular cues present in neurogenic niches of the embryonic forebrain. Our study identified SHH and FGF15 signaling as key pathways that must be tightly modulated to ensure successful differentiation of neocortical progenitors into distinct excitatory neuron lineages in the course of corticogenesis (Fig. 8). These findings further underscore the importance of key modulatory factors at crucial time points in establishing and maintaining neocortical progenitor programs.

\section{Neuronal lineage progression relies on the molecular makeup of the neurogenic niche at E12.5}

Embryonic neocortical progenitors must follow strict lineage programs throughout corticogenesis to ensure the production of molecularly and functionally diverse excitatory neurons in the mature neocortex. Here, we determined that molecular events in early corticogenesis ensure proper lineage progression of neocortical progenitors. This process relies on tightly inhibiting $\mathrm{SHH}$ signaling activity. While pallial-specific progenitors, such as $\mathrm{Pax}^{+}{ }^{+}$RGCs or Tbr2 ${ }^{+}$IPCs, are produced in the Sufu-cKO neocortex, these progenitors possessed underlying transcriptomic changes compromising their lineage progression. Many of these changes involved extracellular or plasma membrane-bound proteins, which can substantially alter intercellular and extracellular interactions within the neurogenic niche. Further, we found that subpallial-specific gene transcripts were already ectopically expressed in the E12.5 Sufu-cKO. These indicated that while proteins encoded by these genes may not be detected in neocortical progenitors at E12.5, the stage has been set to alter their fates. Ultimately, these early alterations can permanently deviate lineage programs of neocortical progenitors, resulting in the production of misspecified excitatory neurons in the postnatal neocortex of Sufu-cKO mice.

\section{SHH signaling alters IPC production by preventing lineage progression of RGCs}

IPC production is especially vulnerable to varying activity levels of $\mathrm{SHH}$ signaling. High $\mathrm{SHH}$ signaling activity inhibits IPC production at early stages of corticogenesis but promotes IPC production at later stages (Shikata et al., 2011; H. Wang et al., 2011; Yabut et al., 2015, 2016; L. Wang et al., 2016). While these studies established the mitogenic effect of SHH signaling to control neocortical progenitor proliferation, including IPCs, a growing number of studies are beginning to identify a role for $\mathrm{SHH}$ signaling in altering progenitor specification as a parallel means of controlling IPC production. SHH signaling partly mediates this effect via Gli3R activity, which when absent or reduced, can sig nificantly impair specification of neocortical progenitors into distinct excitatory neuron subtypes $(\mathrm{H}$. Wang et al., 2011; Yabut et al., 2015; HasenpuschTheil et al., 2018). Supporting these findings, we found upregulated expression of known Gli3R gene targets in the E12.5 Sufu-cKO neocortex, such as FGF15, in the progenitor-rich VZ/SVZ where Gli3 is typically highly expressed particularly in RGCs (Rash and Grove, 2007; Pollen et al., 2014; Hasenpusch-Theil et al., 2015; Kim et al., 2018). Specifically, we found an increase in SHH signaling activation (as detected through an increase in PTCH1 expression) correlating with an increase in FGF15 expression in RGCs lining the VZ. We found a uniform increase in ectopic FGF15 expression in the E12.5 Sufu-cKO neocortex. Despite this, RGCs lining the VZ exhibited variable proliferation rates (Yabut et al., 2015), indicating that FGF15 did not have a consistent effect on progenitor proliferation across the neocortical expanse. Rather, we consistently found that the increase in FGF15 levels, in the Sufu-cKO embryonic neocortex or when added in cultured forebrain slices, inhibited the specification of RGCs into bona fide pallial IPCs. Together, our findings expand evidence of the importance of modulating $\mathrm{SHH}$ signaling in RGCs, particularly via Gli3 activity, in ensuring lineage progression toward the specification of IPCs into distinct excitatory neuron subtypes.

\section{SHH signaling requires FGF signaling to alter neuronal} lineage progression of neocortical progenitors

Our findings revealed that SHH signaling activated FGF signaling leading to disruption of neocortical progenitor specification. Particularly, an increase in FGF15 expression was sufficient to drive these defects. Previous studies showed that FGF15 functions to control progenitor proliferation and differentiation (Borello et al., 2008; Wilson et al., 2012). Supporting a role for FGF15 in proliferation, we found that increased FGF15 expression correlated with the elongation of the E12.5 Sufu-cKO 


\section{Early Corticogenesis (E12.5)}

\section{Controlled SHH and FGF signaling

\author{
maintenance of \\ neocortical progenitor \\ identity
}

\section{Uncontrolled SHH and FGF signaling}

altered neocortical
progenitor
identity
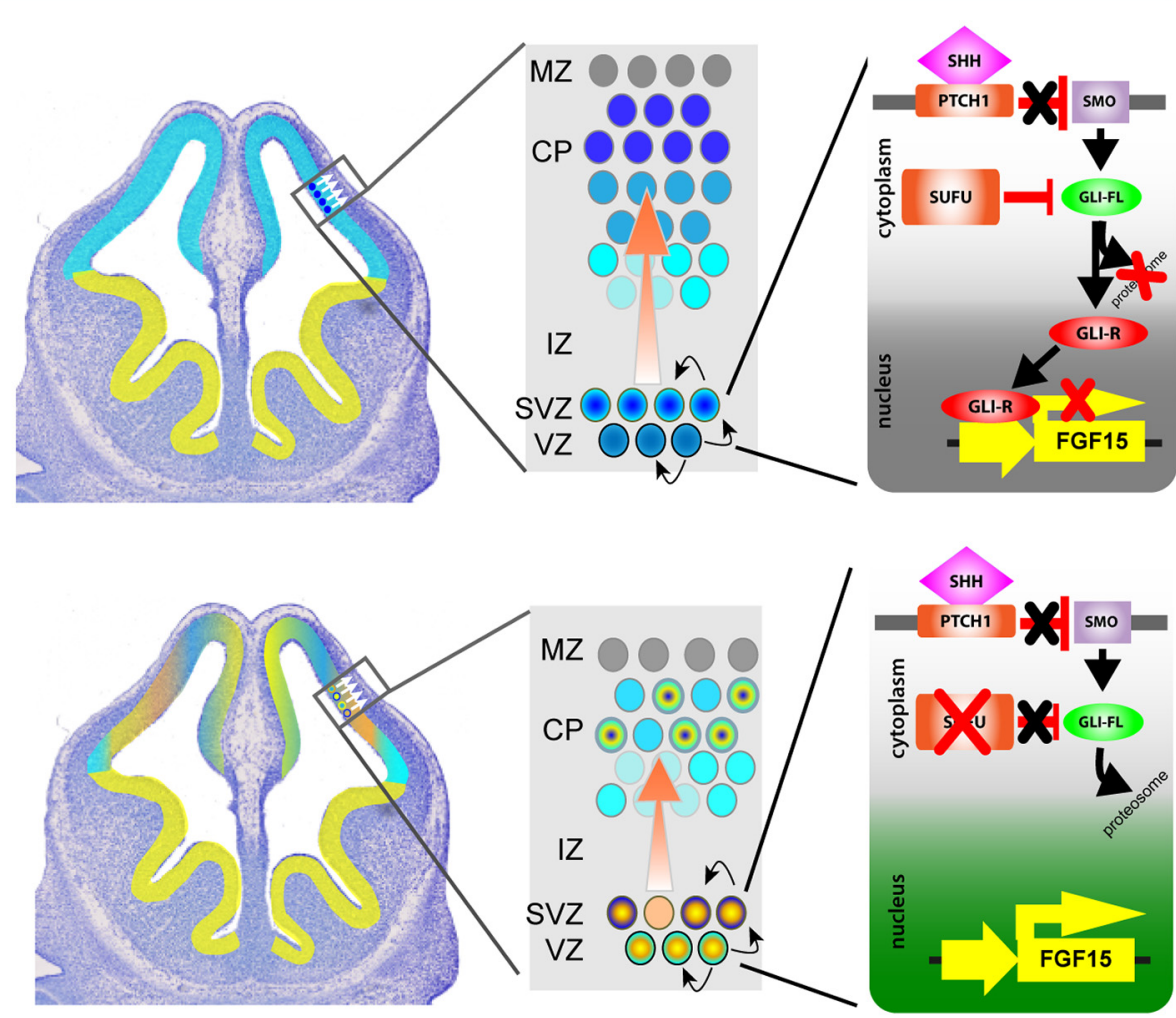

Figure 8. Schematic diagram of how modulation of SHH and FGF signaling affects neocortical progenitors at early stages of corticogenesis. Controlled SHH and FGF signaling, resulting from the repressive function of Gli3R, in the dorsal forebrain, maintains the neocortical identity and specification program of progenitors. Uncontrolled SHH and FGF signaling, achieved when Sufu expression is lost consequently leading to Gli3 degradation and the eventual upregulation of FGF15 expression, results in the inability of neocortical progenitors to maintain the dorsal forebrain identity and specification program.

neocortex. However, as mentioned, FGF15 upregulation did not uniformly correlate with proliferation defects. Supporting this, we found variable levels of activation in MAPK signaling, the downstream intracellular FGF signaling effector. MAPK signaling activity was distinctly higher in dorsal and dorsolateral regions of the E12.5 Sufu-cKO neocortex, but not in dorsomedial regions where FGF15 was also ectopically expressed. Additionally, clonally related progenitors in the rostral neocortical regions of the E12.5 Sufu-cKO neocortex appeared to be uniquely affected. Columnar patterns in the expression levels of Pax6, Olig2, and MAPK activity levels are apparent. These observations suggest that FGF15 differentially affects molecularly distinct neocortical progenitors, which may be predicted by their spatial and temporal localization in the embryonic neocortex. These varying effects may result in changes to cell cycle length, neuronal differentiation, or their specification programs.

While it may be logical to assume that FGF15 will predictably bind to progenitors expressing its cognate receptor, FGFR4, FGFR4 has not been detected in the E12.5 neocortex (Harmer et al., 2004; Tole et al., 2006; Zhang et al., 2006; Borello et al., 2008). On the other hand, FGFR1-3 are expressed in varying levels and spatial domains across the E12.5 neocortical wall and may be sensitive to high levels of FGF15 (Tole et al., 2006; Borello et al., 2008). Alternatively, global changes in the molecular make-up of the E12.5 SufucKO neurogenic niche may have facilitated unique FGF15 interactions. This may explain why Sufu-cKO mice do not completely phenocopy mouse mutants carrying conditional Gli3R mutations (H. Wang et al., 2011; Hasenpusch-Theil et al., 2018). Together, these findings underscore the complexity of FGF15 function in the developing neocortex. Future studies require further elucidation of the molecular properties of FGF15-responsive neocortical progenitors and the signaling pathways transduced to elicit changes in progenitor behavior.

In conclusion, along with the expansion and diversification of neocortical neuron subtypes in the developing brain, anomalies in the production of diverse excitatory neurons underlie a number of neuropsychiatric and neurodevelopmental disorders. The activation of FGF and MAPK signaling cascades, in response to $\mathrm{SHH}$ signaling activation, indicates important potential implications of uncontrolled $\mathrm{SHH}$ signaling in these disorders. For instance, it is evident that abnormal numbers and specification of neuronal subtypes lead to aberrant circuits in autism spectrum disorders (Kaushik and Zarbalis, 2016). High serum levels of SHH and deregulated FGF signaling activity at developmental stages has been implicated in these defects (Vaccarino et al., 2009; Rubenstein, 2011; Al-Ayadhi, 2012; Halepoto et al., 2015). Activation of SHH signaling at early stages of corticogenesis, consequently driving FGF signaling, may profoundly alter the molecular landscape of neocortical progenitors and their progenies. Thus, further investigation of how pathogenic SHH and FGF signaling converge to produce aberrant neuronal subtypes and neocortical circuitry could lay the 
foundation toward detecting, treating, or even reversing the neocortical abnormalities present in neurodevelopmental disorders.

\section{References}

Al-Ayadhi LY (2012) Relationship between sonic hedgehog protein, brainderived neurotrophic factor and oxidative stress in autism spectrum disorders. Neurochem Res 37:394-400.

Beattie R, Hippenmeyer S (2017) Mechanisms of radial glia progenitor cell lineage progression. FEBS Lett 591:3993-4008.

Borello U, Cobos I, Long JE, McWhirter JR, Murre C, Rubenstein JL (2008) FGF15 promotes neurogenesis and opposes FGF8 function during neocortical development. Neural Dev 3:17.

Dave RK, Ellis T, Toumpas MC, Robson JP, Julian E, Adolphe C, Bartlett PF, Cooper HM, Reynolds BA, Wainwright BJ (2011) Sonic hedgehog and notch signaling can cooperate to regulate neurogenic divisions of neocortical progenitors. PLoS One 6:e14680.

Fotaki V, Yu T, Zaki PA, Mason JO, Price DJ (2006) Abnormal positioning of diencephalic cell types in neocortical tissue in the dorsal telencephalon of mice lacking functional Gli3. J Neurosci 26:9282-9292.

Gimeno L, Martinez S (2007) Expression of chickFgf19 and mouseFgf15 orthologs is regulated in the developing brain byFgf8 andShh. Dev Dyn 236:2285-2297.

Guillemot F, Zimmer C (2011) From cradle to grave: the multiple roles of fibroblast growth factors in neural development. Neuron 71:574-588.

Halepoto DM, Bashir S, Zeina R, Al-Ayadhi LY (2015) Correlation between hedgehog $(\mathrm{Hh})$ protein family and brain-derived neurotrophic factor (BDNF) in autism spectrum disorder (ASD). J Coll Physicians Surg Pak 25:882-885

Harmer NJ, Pellegrini L, Chirgadze D, Fernandez-Recio J, Blundell TL (2004) The crystal structure of fibroblast growth factor (FGF) 19 reveals novel features of the FGF family and offers a structural basis for its unusual receptor affinity. Biochemistry 43:629-640.

Hasenpusch-Theil K, Watson JA, Theil T (2015) Direct interactions between Gli3, Wnt8b, and Fgfs underlie patterning of the dorsal telencephalon. Cereb Cortex 27:1137-1148.

Hasenpusch-Theil K, West S, Kelman A, Kozic Z, Horrocks S, McMahon AP, Price DJ, Mason JO, Theil T (2018) Gli3 controls the onset of cortical neurogenesis by regulating the radial glial cell cycle through Cdk6 expression. Development 145:dev163147.

Hevner RF (2019) Intermediate progenitors and Tbr2 in cortical development. J Anat 235:616-625.

Huang DW, Sherman BT, Tan Q, Collins JR, Alvord WG, Roayaei J, Stephens R, Baseler MW, Lane HC, Lempicki RA (2007) The DAVID Gene Functional Classification Tool: a novel biological module-centric algorithm to functionally analyze large gene lists. Genome Biol 8:R183.

Huang da W, Sherman BT, Lempicki RA (2009) Systematic and integrative analysis of large gene lists using DAVID bioinformatics resources. Nat Protoc 4:44-57.

Kaushik G, Zarbalis KS (2016) Prenatal neurogenesis in autism spectrum disorders. Front Chem 4:12.

Kim JJ, Jiwani T, Erwood S, Loree J, Rosenblum ND (2018) Suppressor of fused controls cerebellar neuronal differentiation in a manner modulated by GLI3 repressor and Fgf15. Dev Dyn 247:156-169.

Komada M, Saitsu H, Kinboshi M, Miura T, Shiota K, Ishibashi M (2008a) Hedgehog signaling is involved in development of the neocortex. Development 135:2717-2727.

Komada M, Saitsu H, Shiota K, Ishibashi M (2008b) Expression of Fgf15 is regulated by both activator and repressor forms of Gli2 in vitro. Biochem Biophys Res Commun 369:350-356.

Long F, Zhang XM, Karp S, Yang Y, McMahon AP (2001) Genetic manipulation of hedgehog signaling in the endochondral skeleton reveals a direct role in the regulation of chondrocyte proliferation. Development 128:5099-5108.

Miyoshi G, Butt SJ, Takebayashi H, Fishell G (2007) Physiologically distinct temporal cohorts of cortical interneurons arise from telencephalic Olig2expressing precursors. J Neurosci 27:7786-7798.
Palma V, Ruiz i Altaba A (2004) Hedgehog-GLI signaling regulates the behavior of cells with stem cell properties in the developing neocortex. Development 131:337-345.

Petryniak MA, Potter GB, Rowitch DH, Rubenstein JL (2007) Dlx1 and Dlx2 control neuronal versus oligodendroglial cell fate acquisition in the developing forebrain. Neuron 55:417-433.

Pollen AA, Nowakowski TJ, Shuga J, Wang X, Leyrat AA, Lui JH, Li N, Szpankowski L, Fowler B, Chen P, Ramalingam N, Sun G, Thu M, Norris M, Lebofsky R, Toppani D, Kemp DW, Wong M, Clerkson B, Jones BN, et al. (2014) Low-coverage single-cell mRNA sequencing reveals cellular heterogeneity and activated signaling pathways in developing cerebral cortex. Nat Biotechnol 32:1053-1058.

Pospisilik JA, Schramek D, Schnidar H, Cronin SJF, Nehme NT, Zhang X, Knauf C, Cani PD, Aumayr K, Todoric J, Bayer M, Haschemi A, Puviindran V, Tar K, Orthofer M, Neely GG, Dietzl G, Manoukian A, Funovics M, Prager G, et al. (2010) Drosophila genome-wide obesity screen reveals hedgehog as a determinant of brown versus white adipose cell fate. Cell 140:148-160.

Rash BG, Grove EA (2007) Patterning the dorsal telencephalon: a role for sonic hedgehog? J Neurosci 27:11595-11603.

Rubenstein JL (2011) Annual Research Review: development of the cerebral cortex: implications for neurodevelopmental disorders. J Child Psychol Psychiatry 52:339-355.

Shikata Y, Okada T, Hashimoto M, Ellis T, Matsumaru D, Shiroishi T, Ogawa M, Wainwright B, Motoyama J (2011) Ptch1-mediated dosage-dependent action of Shh signaling regulates neural progenitor development at late gestational stages. Dev Biol 349:147-159.

Siegenthaler JA, Ashique AM, Zarbalis K, Patterson KP, Hecht JH, Kane MA, Folias AE, Choe Y, May SR, Kume T, Napoli JL, Peterson AS, Pleasure SJ (2009) Retinoic acid from the meninges regulates cortical neuron generation. Cell 139:597-609.

Sohal VS, Rubenstein JL (2019) Excitation-inhibition balance as a framework for investigating mechanisms in neuropsychiatric disorders. Mol Psychiatry 24:1248-1257.

Tole S, Gutin G, Bhatnagar L, Remedios R, Hébert JM (2006) Development of midline cell types and commissural axon tracts requires Fgfrl in the cerebrum. Dev Biol 289:141-151.

Toresson H, Potter SS, Campbell K (2000) Genetic control of dorsal-ventral identity in the telencephalon: opposing roles for Pax6 and Gsh2. Development 127:4361-4371.

Vaccarino FM, Grigorenko EL, Smith KM, Stevens HE (2009) Regulation of cerebral cortical size and neuron number by fibroblast growth factors: implications for autism. J Autism Dev Disord 39:511-520.

Wang H, Ge G, Uchida Y, Luu B, Ahn S (2011) Gli3 is required for maintenance and fate specification of cortical progenitors. J Neurosci 31:64406448.

Wang L, Hou S, Han YG (2016) Hedgehog signaling promotes basal progenitor expansion and the growth and folding of the neocortex. Nat Neurosci 19:888-896.

Wang Y, Kim E, Wang X, Novitch BG, Yoshikawa K, Chang LS, Zhu Y (2012) ERK inhibition rescues defects in fate specification of Nf1-deficient neural progenitors and brain abnormalities. Cell 150:816-830.

Wilson SL, Wilson JP, Wang C, Wang B, McConnell SK (2012) Primary cilia and Gli3 activity regulate cerebral cortical size. Dev Neurobiol 72:11961212.

Yabut OR, Fernandez G, Huynh T, Yoon K, Pleasure SJ (2015) Suppressor of fused is critical for maintenance of neuronal progenitor identity during corticogenesis. Cell Rep 12:2021-2034.

Yabut OR, Ng H, Fernandez G, Yoon K, Kuhn J, Pleasure SJ (2016) Loss of suppressor of fused in mid-corticogenesis leads to the expansion of intermediate progenitors. J Dev Biol 4:29.

Ypsilanti AR, Rubenstein JL (2016) Transcriptional and epigenetic mechanisms of early cortical development: an examination of how Pax6 coordinates cortical development. J Comp Neurol 524:609-629.

Zhang X, Ibrahimi OA, Olsen SK, Umemori H, Mohammadi M, Ornitz DM (2006) Receptor specificity of the fibroblast growth factor family: the complete mammalian FGF family. J Biol Chem 281:15694-15700. 